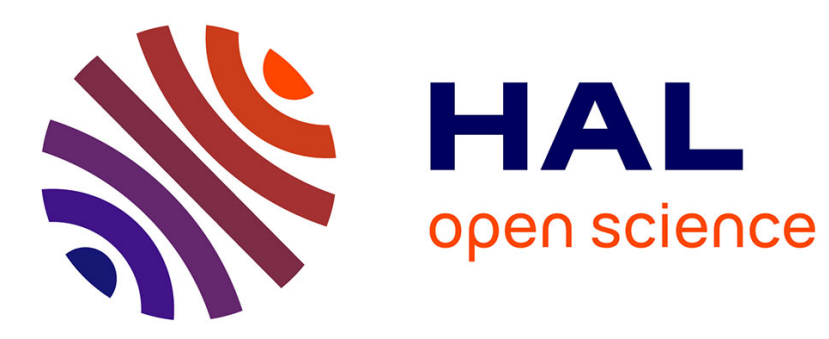

\title{
Hierarchical Beam Finite Elements Based Upon a Variables Separation Method
}

G. Giunta, S. Belouettar, O. Polit, L. Gallimard, P. Vidal, M. d'Ottavio

\section{To cite this version:}

G. Giunta, S. Belouettar, O. Polit, L. Gallimard, P. Vidal, et al.. Hierarchical Beam Finite Elements Based Upon a Variables Separation Method. International Journal of Applied Mechanics, 2016, 8 (2), pp.1650026. 10.1142/S1758825116500265 . hal-01367029

\section{HAL Id: hal-01367029 \\ https://hal.science/hal-01367029}

Submitted on 5 Jan 2018

HAL is a multi-disciplinary open access archive for the deposit and dissemination of scientific research documents, whether they are published or not. The documents may come from teaching and research institutions in France or abroad, or from public or private research centers.
L'archive ouverte pluridisciplinaire HAL, est destinée au dépôt et à la diffusion de documents scientifiques de niveau recherche, publiés ou non, émanant des établissements d'enseignement et de recherche français ou étrangers, des laboratoires publics ou privés. 


\title{
Hierarchical Beam Finite Elements Based Upon a Variables Separation Method
}

\author{
Gaetano Giunta* and Salim Belouettar \\ Luxembourg Institute of Science and Technology \\ 5, Avenue des Hauts-Fourneaux \\ L-4362 Esch-sur-Alzette, Luxembourg \\ *gaetano.giunta@list.lu \\ Olivier Polit, Laurent Gallimard, Philippe Vidal \\ and Michele D'ottavio \\ Université Paris Ouest Nanterre-La Défense \\ 50 Rue de Sèvres, 92410 Ville d'Avray, France \\ Received 13 November 2015 \\ Revised 13 January 2016 \\ Accepted 16 January 2016 \\ Published 4 April 2016
}

\begin{abstract}
A family of hierarchical one-dimensional beam finite elements developed within a variables separation framework is presented. A Proper Generalized Decomposition (PGD) is used to divide the global three-dimensional problem into two coupled ones: one defined on the cross-section space (beam modeling kinematic approximation) and one belonging to the axis space (finite element solution). The displacements over the cross-section are approximated via a Unified Formulation (UF). A Lagrangian approximation is used along the beam axis. The resulting problems size is smaller than that of the classical equivalent finite element solution. The approach is, then, particularly attractive for higherorder beam models and refined axial meshes. The numerical investigations show that the proposed method yields accurate yet computationally affordable three-dimensional displacement and stress fields solutions.
\end{abstract}

Keywords: Three-dimensional beam structures; one-dimensional hierarchical modeling; finite element modeling; proper generalized decomposition.

\section{Introduction}

Beam models are used in several engineering fields, such as aeronautics, space, automotive and civil construction to model many structural elements where one dimension is predominant when compared to the other ones. Aircraft wings, helicopter rotor blades, robot arms are just few examples of problems that can be studied by means of a beam theory [Hodges, 1990]. Accurate yet efficient models able to capture higher-order mechanical effects are needed in order to design and optimize this type of structures. Beam modeling, therefore, is an important and up-to-date research topic. 
Euler-Bernoulli's and Timoshenko's theories represent the classical approach towards the bending analysis of beam structures. Within these theories, the crosssection is supposed to be rigid on its own plane. Euler-Bernoulli's theory discards the shear deformation, whereas Timoshenko's model accounts for a constant value over the cross-section. Furthermore, material stiffness coefficients should be opportunely reduced in order to contrast the Poisson locking [Giunta et al., 2013a, $2013 \mathrm{~b}]$.

As far as torsional problems are concerned, Saint-Venant's and Prandtl's models represent the classical solutions. Saint-Venant postulated the displacement field assuming that the deformation of a twisted shaft consists in a rigid rotation, as in the case of a circular cross-section, and a warping that is constant along the beam axis. The membrane analogy for the study of torsion was used by Prandtl.

Improvements in classical beam models have been proposed over the last years to account for nonclassical effects and nonconventional materials. Some of them are discussed hereafter. Kapania and Raciti [1989a, 1989b] presented a literature review about static, buckling, free-vibration and wave propagation analysis of beams. Stephen and Levinson [1979] accounted for a linear variation of the shear deformation over the cross-section. Within the framework of the finite element method, Kant and Manjunath [1989] proposed two kinematic fields with a second- and a third-order variation of the axial displacement, respectively. Rand [1998] formulated a five degrees of freedom (dof) model: three cross-sectional displacements and a twist angle, which are constant above the cross-section and a three-dimensional warping function. Ganapathi et al. [1999] accounted for transverse shear and warping in sandwich beams by means of sinus refined finite elements. Stemming from that work, Vidal and Polit [2008] developed a three-node finite element for the study of laminated beams. An analytical model of I-shaped composite beams accounting for flexural-torsional mechanics was proposed in Lee and Lee [2004]. That model was, then, enhanced by incorporating the transverse shear deformation [Lee, 2005]. Stemming from a work by Berdichevsky et al. [1992], a variational-asymptotic approach for the Timoshenko-like modeling of initially curved and twisted composite beams was used by Yu et al. [2002]. Thanks to that approach, a priori assumptions over the cross-section are not imposed whilst the general three-dimensional anisotropic elasticity problem is decoupled into a linear two-dimensional cross-section analysis (in order to obtain the cross-section stiffness matrix) and a nonlinear one-dimensional beam analysis. A Timoshenko-like model was, then, obtained by reproducing the three-dimensional strain energy in terms of one-dimensional strains. Classical Timoshenko's model is enhanced in the sense that in- and out-of-plane warping are accounted for.

Within this paper, the derivation of accurate yet computationally efficient finite elements for the static analysis of three-dimensional beam structures is proposed. The kinematic field is axiomatically assumed over the cross-section via a Unified Formulation (UF). This UF was previously derived for plates and shells ([Carrera, 
2003, Carrera and Giunta, 2009a and 2009b] and [Giunta et al., 2011]) and, then, extended to the analysis of beam structures, [Carrera and Giunta, 2010, Carrera et al., 2010 and 2011] and [Giunta et al., 2010a, 2010b, 2011, 2012 and 2013]. This approach is attractive since accurate higher-order models can be straightforwardly obtained. The weak form of the governing differential equations is derived through the Principle of Virtual Displacement (PVD) in terms of a "fundamental nucleus" that does not depend upon the displacement field approximation order over the cross-section nor the number of nodes per element along the beam axis. Displacement-based theories accounting for nonclassical effects, such as transverse shear and cross-section in- and out-of-plane warping, are straightforwardly derived.

As a matter of fact, the computer memory needed for the analysis can become prohibitive as the number of elements and approximation order increase. The use of an "out-of-core" computation approach where part of the static memory of the hard disk is also used [Castellanos and Larrazabal, 2013] is a possible strategy to overcome stack overflow. A shortcoming of this approach is that hard disk memory is slow to be accessed to. In this paper, a Proper Generalized Decomposition (PGD) is used to reduce the need in memory and perform the calculation all "in-core" by decoupling the approximation over the beam cross-section from that along the axis and solving two coupled nonlinear systems of considerably smaller size than that of a corresponding classical finite element solution. The used approach belongs to the framework of model reduction [Ammar et al., 2006]. A detailed review of the PGD and its application in different engineering fields can be found in Nouy [2010] and Chinesta et al. [2011]. To the best of authors' knowledge, one of the first application to structural mechanics can be found in Savoia and Reddy [1992] and, more recently, in Bognet et al. [2012]. The PGD has been used to study two-dimensional laminated and sandwich beams via quadratic and fourth-order through-the-thickness approximations by Vidal et al. [2012a and 2012b], laminated and sandwich plates by a fourth-order layer-wise model by Vidal et al. [2013 and 2014a] and cylindrical composite shells in Vidal et al. [2014b]. Within the PGD framework, Gallimard et al. [2011] carried out a reliability analysis in terms of failure probability of multi-layered two-dimensional structures where the Young moduli were assumed as stochastic variables.

For the beam elements proposed in this paper, shear locking is corrected by means of a selective integration strategy. Isotropic slender and short beams are investigated. The proposed models are validated towards solutions obtained via a closed-form Navier-type method as well as corresponding one-dimensional finite elements. Three-dimensional finite element solutions obtained through ANSYS are also considered. Numerical results show that accurate results can be obtained with a reduced computational effort when compared with the corresponding classical one-dimensional finite elements as well as the three-dimensional solutions. 


\section{Preliminaries}

A beam is a structure whose axial extension $(l)$ is predominant with respect to any other dimension orthogonal to it. The cross-section $(\Omega)$ is defined by intersecting the beam with planes orthogonal to its axis. A Cartesian reference system is adopted. The $x$ coordinate is coincident with the axis of the beam and it is bounded such that $0 \leq x \leq l$, whereas the $y$ - and $z$-axes are two orthogonal directions laying on $\Omega$. Figure 1 presents the beam geometry and the reference system.

The displacement field is:

$$
\mathbf{u}^{T}(x, y, z)=\left\{u_{x}(x, y, z) \quad u_{y}(x, y, z) \quad u_{z}(x, y, z)\right\}
$$

where $u_{x}, u_{y}$ and $u_{z}$ are the displacement components along the $x$-, $y$ - and $z$-axis, respectively. Superscript " $T$ " represents the transposition operator.

Stresses $(\boldsymbol{\sigma})$ are grouped into vectors $\boldsymbol{\sigma}_{n} \in \mathbb{R}^{3}$ acting on the cross-section:

$$
\boldsymbol{\sigma}_{n}^{T}=\left\{\begin{array}{lll}
\sigma_{x x} & \sigma_{x y} & \sigma_{x z}
\end{array}\right\}
$$

and $\boldsymbol{\sigma}_{p} \in \mathbb{R}^{3}$ acting on planes orthogonal to $\Omega$ :

$$
\boldsymbol{\sigma}_{p}^{T}=\left\{\begin{array}{lll}
\sigma_{y y} & \sigma_{z z} & \sigma_{y z}
\end{array}\right\}
$$

The associated strains, $\varepsilon_{n}$ and $\varepsilon_{p}$, are:

$$
\varepsilon_{n}^{T}=\left\{\begin{array}{lll}
\varepsilon_{x x} & \gamma_{x y} & \gamma_{x z}
\end{array}\right\}, \quad \varepsilon_{p}^{T}=\left\{\begin{array}{lll}
\varepsilon_{y y} & \varepsilon_{z z} & \gamma_{y z}
\end{array}\right\} .
$$

In the case of small displacements, linear relations between strains and displacements hold:

$$
\varepsilon_{n}^{T}=\left\{u_{x, x} \quad u_{x, y}+u_{y, x} \quad u_{x, z}+u_{z, x}\right\}, \quad \varepsilon_{p}^{T}=\left\{\begin{array}{lll}
u_{y, y} & u_{z, z} \quad u_{y, z}+u_{z, y}
\end{array}\right\} .
$$

Subscripts " $x$ ", " $y$ " and " $z$ ", when preceded by comma, represent derivation versus the corresponding spatial coordinate. Equations (5) in a compact vectorial notation read:

$$
\varepsilon_{n}=\mathbf{D}_{n p} \mathbf{u}+\mathbf{D}_{n x} \mathbf{u}, \quad \varepsilon_{p}=\mathbf{D}_{p} \mathbf{u}
$$

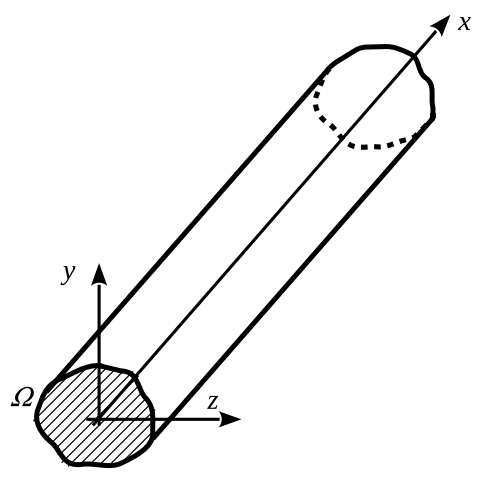

Fig. 1. Beam geometry and reference system. 
$\mathbf{D}_{n p}, \mathbf{D}_{n x}$ and $\mathbf{D}_{p}$ are the following differential matrix operators:

$$
\mathbf{D}_{n p}=\left[\begin{array}{ccc}
0 & 0 & 0 \\
\frac{\partial}{\partial y} & 0 & 0 \\
\frac{\partial}{\partial z} & 0 & 0
\end{array}\right], \quad \mathbf{D}_{n x}=\mathbf{I} \frac{\partial}{\partial x}, \quad \mathbf{D}_{p}=\left[\begin{array}{ccc}
0 & \frac{\partial}{\partial y} & 0 \\
0 & 0 & \frac{\partial}{\partial z} \\
0 & \frac{\partial}{\partial z} & \frac{\partial}{\partial y}
\end{array}\right],
$$

being $\mathbf{I}$ the unit matrix in $\mathbb{R}^{3 \times 3}$.

Under the hypothesis of linear elastic materials, the constitutive relation is:

$$
\boldsymbol{\sigma}_{p}=\mathbf{C}_{p p} \boldsymbol{\varepsilon}_{p}+\mathbf{C}_{p n} \boldsymbol{\varepsilon}_{n}, \quad \boldsymbol{\sigma}_{n}=\mathbf{C}_{n p} \boldsymbol{\varepsilon}_{p}+\mathbf{C}_{n n} \boldsymbol{\varepsilon}_{n} .
$$

In the case of an isotropic material, the stiffness matrices $\mathbf{C}_{p p}, \mathbf{C}_{p n}, \mathbf{C}_{n p}$ and $\mathbf{C}_{n n} \in$ $\mathbb{R}^{3 \times 3}$ are:

$$
\begin{gathered}
\mathbf{C}_{p p}=\left[\begin{array}{ccc}
C_{22} & C_{23} & 0 \\
C_{23} & C_{33} & 0 \\
0 & 0 & C_{44}
\end{array}\right], \quad \mathbf{C}_{p n}=\mathbf{C}_{n p}^{T}=\left[\begin{array}{ccc}
C_{12} & 0 & 0 \\
C_{13} & 0 & 0 \\
0 & 0 & 0
\end{array}\right], \\
\mathbf{C}_{n n}=\left[\begin{array}{ccc}
C_{11} & 0 & 0 \\
0 & C_{66} & 0 \\
0 & 0 & C_{55}
\end{array}\right],
\end{gathered}
$$

with:

$$
\begin{aligned}
& C_{11}=C_{22}=C_{33}=\frac{1-\nu}{(1+\nu)(1-2 \nu)} E, \\
& C_{12}=C_{13}=C_{23}=\frac{\nu}{(1+\nu)(1-2 \nu)} E, \\
& C_{44}=C_{55}=C_{66}=\frac{1}{2(1+\nu)} E,
\end{aligned}
$$

being $E$ the Young modulus and $\nu$ the Poisson ratio.

\section{Hierarchical PGD Beam Elements}

In the framework of the proposed PGD-based unified finite element modeling, the variation of each displacement component versus the spatial coordinates is decomposed into the sum of coupled functions or "couples". Each couple is the product of functions $\mathbf{f}^{i}=\mathbf{f}^{i}(y, z)$ and $\mathbf{v}^{i}=\mathbf{v}^{i}(x)$ :

$$
\begin{aligned}
\mathbf{u}(x, y, z): \mathcal{D}_{\Omega} \times \mathcal{D}_{x} & \rightarrow \mathbb{R}^{3} \\
(y, z, x) & \mapsto \mathbf{f}^{i}(y, z) \circ \mathbf{v}^{i}(x) \quad \text { with } i=1,2, \ldots, N_{c} .
\end{aligned}
$$


$\mathbf{f}^{i}$, which derives from the one-dimensional beam modeling, depends upon the crosssection coordinates $y$ and $z$ only. Function $\mathbf{v}^{i}$ accounts for the variation versus the axial coordinate $x$. This latter, within the beam finite element modeling framework, is obtained via classical shape functions. $N_{c}$ is the number of couples. According to Einstein's notation, a repeated index is a dummy index that, unless otherwise stated, stands for summation. This notation is extensively used through the paper and it allows to derive the problem governing equations in terms of a single "fundamental nucleus" regardless the approximation order over the cross-section $(N)$, the number of nodes per element along the beam axis $\left(N_{n}\right)$ and the number of couples in the PGD approximation. The actual governing equations due to a fixed cross-section approximation order, number of nodes per element along the beam axis and number of couples are obtained straightforwardly via summation of the nucleus corresponding to each term of the expansion. In this sense, $N, N_{n}$ and $N_{c}$ are free parameters of the formulation. The operator "o" in Eq. (11) is Hadamard's element-wise vector product. It is defined as follows [Horn, 1990]:

$$
\mathbf{f}^{i} \circ \mathbf{v}^{i}: \mathbb{R}^{3} \rightarrow \mathbb{R}^{3}\left\{\begin{array}{l}
f_{x}^{i} \\
f_{y}^{i} \\
f_{z}^{i}
\end{array}\right\} \circ\left\{\begin{array}{c}
v_{x}^{i} \\
v_{y}^{i} \\
v_{z}^{i}
\end{array}\right\}=\left\{\begin{array}{l}
f_{x}^{i} v_{x}^{i} \\
f_{y}^{i} v_{y}^{i} \\
f_{z}^{i} v_{z}^{i}
\end{array}\right\} .
$$

It should be noticed that, by its definition, Hadamard's operator is commutative and distributive and it can be regarded as a compact expression for the following matrix-vector product:

$$
\mathbf{f}^{i} \circ \mathbf{v}^{i}=\mathbf{v}^{i} \circ \mathbf{f}^{i}=\left[\begin{array}{ccc}
f_{x}^{i} & 0 & 0 \\
0 & f_{y}^{i} & 0 \\
0 & 0 & f_{z}^{i}
\end{array}\right]\left\{\begin{array}{c}
v_{x}^{i} \\
v_{y}^{i} \\
v_{z}^{i}
\end{array}\right\}=\left[\begin{array}{ccc}
v_{x}^{i} & 0 & 0 \\
0 & v_{y}^{i} & 0 \\
0 & 0 & v_{z}^{i}
\end{array}\right]\left\{\begin{array}{c}
f_{x}^{i} \\
f_{y}^{i} \\
f_{z}^{i}
\end{array}\right\} .
$$

Although the two notations are equivalent, in the theoretical developments Hadamard's product is mainly used. The matrix notation is introduced when matrix multiplication has to be done on a term in the Hadamard product.

The following approximations over the beam cross-section and its axis are introduced:

$$
\begin{aligned}
\mathbf{f}^{i}(y, z)=F_{\tau}(y, z) \mathbf{q}_{\mathcal{D}_{\Omega} \tau}^{i} & \text { with } \tau=1,2, \ldots, N_{u}, \\
\mathbf{v}^{i}(x)=N_{j}(x) \mathbf{q}_{\mathcal{D}_{x} j}^{i} & \text { with } j=1,2, \ldots, N_{n} .
\end{aligned}
$$

$F_{\tau}(y, z)$ are the a priori approximating functions over the beam cross-section. Within this work, they are MacLaurin's polynomials. The dummy index $\tau$ ranges over the number of cross-section approximation terms $N_{u}$. It depends upon the cross-section approximation order $N$ as follows:

$$
N_{u}=\frac{(N+1)(N+2)}{2} .
$$

$N_{u}$ and $F_{\tau}$ as functions of $N$ can be obtained through Pascal's triangle as shown in Table $1 . N_{j}(x)$ are the classical Lagrangian finite element shape functions. They 
Table 1. MacLaurin's polynomials via Pascal's triangle.

\begin{tabular}{|c|c|c|}
\hline$N$ & $N_{u}$ & $F_{\tau}$ \\
\hline 0 & 1 & $F_{1}=1$ \\
\hline 1 & 3 & $F_{2}=y \quad F_{3}=z$ \\
\hline 2 & 6 & $F_{4}=y^{2} \quad F_{5}=y z \quad F_{6}=z^{2}$ \\
\hline 3 & 10 & $F_{7}=y^{3} \quad F_{8}=y^{2} z \quad F_{9}=y z^{2} \quad F_{10}=z^{3}$ \\
\hline$\vdots$ & $\vdots$ & $\vdots$ \\
\hline$N$ & $\frac{(N+1)(N+2)}{2}$ & $F_{\frac{\left(N^{2}+N+2\right)}{2}}=y^{N} \quad F_{\frac{\left(N^{2}+N+4\right)}{2}}=y^{N-1} z \quad \cdots \quad F_{\frac{N(N+3)}{2}}=y z^{N-1} \quad F_{\frac{(N+1)(N+2)}{2}}=z^{N}$ \\
\hline
\end{tabular}

approximate the displacements along the beam axis in a $C^{0}$ sense up to an order $N_{n}-1$. The dummy index $j$ ranges over the number of nodes per element. Linear, quadratic and cubic elements along the beam axis are considered. These elements are addressed by "B2", "B3" and "B4", respectively. For the sake of simplicity, their shape functions are not reported here. They can be found in Bathe [1996]. $\mathbf{q}_{\mathcal{D}_{\Omega} \tau}^{i}$ and $\mathbf{q}_{\mathcal{D}_{x} j}^{i}$ are the unknowns for the cross-section $\left(\mathcal{D}_{\Omega}\right)$ and axis $\left(\mathcal{D}_{x}\right)$ approximation spaces, respectively. By replacing Eqs. (14) within Eq. (11), the displacement field becomes:

$$
\mathbf{u}(x, y, z)=F_{\tau}(y, z) N_{j}(x) \mathbf{q}_{\mathcal{D}_{\Omega} \tau}^{i} \circ \mathbf{q}_{\mathcal{D}_{x} j}^{i}
$$

Within a PGD framework, a stiffness matrix for each approximation space is derived. Thanks to the UF, these matrices are obtained in a nuclear form via the weak form of the PVDs:

$$
\delta \mathscr{L}_{\text {int }}^{e}-\delta \mathscr{L}_{\text {ext }}^{e}=0
$$

where $\delta$ represents a virtual variation, $\mathscr{L}_{\text {int }}^{e}$ the strain energy and $\mathscr{L}_{\text {ext }}^{e}$ the work done by the external loads. Using Eq. (16), the virtual variation of the displacement reads:

$$
\delta \mathbf{u}^{T}=\left(\delta \mathbf{q}_{\mathcal{D}_{\Omega} \sigma}^{s T} \circ \mathbf{q}_{\mathcal{D}_{x} l}^{s}+\delta \mathbf{q}_{\mathcal{D}_{x} l}^{s T} \circ \mathbf{q}_{\mathcal{D}_{\Omega} \sigma}^{s}\right) F_{\sigma} N_{l} .
$$

By recalling Eq. (13), Eq. (18) is rewritten using Hadamard's product matrix notation:

$$
\delta \mathbf{u}^{T}=\left(\delta \mathbf{q}_{\mathcal{D}_{\Omega} \sigma}^{s T} \mathbf{Q}_{\mathcal{D}_{x} l}^{s}+\delta \mathbf{q}_{\mathcal{D}_{x} l}^{s T} \mathbf{Q}_{\mathcal{D}_{\Omega} \sigma}^{s}\right) F_{\sigma} N_{l}
$$

where $\mathbf{Q}_{\mathcal{D}_{x} l}^{s}$ and $\mathbf{Q}_{\mathcal{D}_{\Omega} \sigma}^{s}$ are the following diagonal matrices:

$$
\mathbf{Q}_{\mathcal{D}_{x} l}^{s}=\left[\begin{array}{ccc}
q_{\mathcal{D}_{x} l x}^{s} & 0 & 0 \\
0 & q_{\mathcal{D}_{x} l y}^{s} & 0 \\
0 & 0 & q_{\mathcal{D}_{x} l z}^{s}
\end{array}\right], \quad \mathbf{Q}_{\mathcal{D}_{\Omega} \sigma}^{s}=\left[\begin{array}{ccc}
q_{\mathcal{D}_{\Omega} \sigma x}^{s} & 0 & 0 \\
0 & q_{\mathcal{D}_{\Omega} \sigma y}^{s} & 0 \\
0 & 0 & q_{\mathcal{D}_{\Omega} \sigma z}^{s}
\end{array}\right]
$$

containing the problems unknowns of each approximation space. 


\subsection{Virtual variation of the strain energy}

Coherently with the stresses and strains grouping in Eqs. (2) and (3), the virtual variation of the internal work reads:

$$
\delta \mathscr{L}_{\text {int }}^{e}=\int_{V_{e}}\left(\delta \varepsilon_{p}^{T} \boldsymbol{\sigma}_{p}+\delta \varepsilon_{n}^{T} \boldsymbol{\sigma}_{n}\right) d V,
$$

where $V_{e}$ stands for the volume of an element:

$$
V_{e}=\Omega_{e} \times l_{e},
$$

being $l_{e}$ the element axial length and $\Omega_{e}$ the element cross-section.

The virtual variation of the strains accounting for the displacement approximation in Eq. (16) is:

$$
\begin{aligned}
& \delta \varepsilon_{n}=\left(\delta \mathbf{q}_{\mathcal{D}_{\Omega} \sigma}^{s T} \mathbf{Q}_{\mathcal{D}_{x} l}^{s}+\delta \mathbf{q}_{\mathcal{D}_{x} l}^{s T} \mathbf{Q}_{\mathcal{D}_{\Omega} \sigma}^{s}\right)\left[N_{l}\left(\mathbf{D}_{n p} F_{\sigma} \mathbf{I}\right)^{T}+F_{\sigma} N_{l, x} \mathbf{I}\right], \\
& \delta \varepsilon_{p}=\left(\delta \mathbf{q}_{\mathcal{D}_{\Omega} \sigma}^{s T} \mathbf{Q}_{\mathcal{D}_{x} l}^{s}+\delta \mathbf{q}_{\mathcal{D}_{x} l}^{s T} \mathbf{Q}_{\mathcal{D}_{\Omega} \sigma}^{s}\right) N_{l}\left(\mathbf{D}_{p} F_{\sigma} \mathbf{I}\right)^{T} .
\end{aligned}
$$

After substitution of the constitutive equations, Eqs. (8), the geometric relations, Eqs. (6) and (23), and the displacement approximation, Eqs. (16), the virtual variation of the element strain energy becomes:

$$
\delta \mathscr{L}_{\text {int }}^{e}=\delta \mathbf{q}_{\mathcal{D}_{\Omega} \sigma}^{s T} \mathbf{K}_{\mathcal{D}_{x}}^{\sigma \tau s i} \mathbf{q}_{\mathcal{D}_{\Omega} \tau}^{i}+\delta \mathbf{q}_{\mathcal{D}_{x} l}^{s T} \mathbf{K}_{\mathcal{D}_{\Omega}}^{l j s i} \mathbf{q}_{\mathcal{D}_{x} j}^{i},
$$

where:

$$
\mathbf{K}_{\mathcal{D}_{x}}^{\sigma \tau s i}=\mathbf{Q}_{\mathcal{D}_{x} l}^{s} \mathbf{K}^{l j \sigma \tau} \mathbf{Q}_{\mathcal{D}_{x} j}^{i}, \quad \mathbf{K}_{\mathcal{D}_{\Omega}}^{l j s i}=\mathbf{Q}_{\mathcal{D}_{\Omega} \sigma}^{s} \mathbf{K}^{l j \sigma \tau} \mathbf{Q}_{\mathcal{D}_{\Omega} \tau}^{i} .
$$

$\mathbf{K}_{\mathcal{D}_{x}}^{\sigma \tau s i}$ and $\mathbf{K}_{\mathcal{D}_{\Omega}}^{l j s i}$ are the projection of $\mathbf{K}^{l j \sigma \tau}$ over the space $\mathcal{D}_{x}$ and $\mathcal{D}_{\Omega}$, respectively. $\mathbf{K}^{l j \sigma \tau} \in \mathbb{R}^{3 \times 3}$ is the fundamental nucleus of the element stiffness matrix as derived from a classical finite element approach [Carrera et al., 2010 and 2011]:

$$
\begin{aligned}
\mathbf{K}^{l j \sigma \tau}= & \int_{l_{e}} \int_{\Omega_{e}}\left\{[ N _ { l } ( \mathbf { D } _ { n p } F _ { \sigma } ) ^ { T } + N _ { l , x } F _ { \sigma } \mathbf { I } ] \left[N_{j} \mathbf{C}_{n p}\left(\mathbf{D}_{p} F_{\tau}\right)\right.\right. \\
& \left.+N_{j} \mathbf{C}_{n n}\left(\mathbf{D}_{n p} F_{\tau}\right)+N_{j, x} F_{\tau} \mathbf{C}_{n n}\right]+N_{l}\left(\mathbf{D}_{p} F_{\sigma}\right)^{T}\left[N_{j} \mathbf{C}_{p p}\left(\mathbf{D}_{p} F_{\tau}\right)\right. \\
& \left.\left.+N_{j} \mathbf{C}_{p n}\left(\mathbf{D}_{n p} F_{\tau}\right)+N_{j, x} F_{\tau} \mathbf{C}_{p n}\right]\right\} d \Omega d x .
\end{aligned}
$$

The explicit expression of its components is:

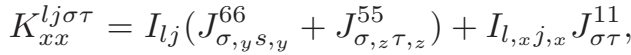

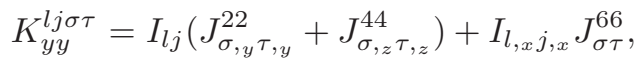

$$
\begin{aligned}
& K_{z z}^{l j \sigma \tau}=I_{l j}\left(J_{\sigma, y}^{44,,_{y}}+J_{\sigma, z \tau, z}^{33}\right)+I_{l,,_{x} j_{x}} J_{\sigma \tau}^{55}, \\
& K_{x y}^{l j \sigma \tau}=I_{l j, x} J_{\sigma, y}^{66}+I_{l, x_{x} j} J_{\sigma \tau, y}^{12}, \\
& K_{y x}^{l j \sigma \tau}=I_{l j, x} J_{\sigma, y}^{12}+I_{l,{ }_{x} j} J_{\sigma \tau, y}^{66},
\end{aligned}
$$




$$
\begin{aligned}
& K_{x z}^{l j \sigma \tau}=I_{l j, x} J_{\sigma, z}^{55}+I_{l,{ }_{x} j} J_{\sigma \tau, z}^{13}, \\
& K_{z x}^{l j \sigma \tau}=I_{l j, x} J_{\sigma,,_{z} \tau}^{13}+I_{l,{ }_{x} j} J_{\sigma \tau, z}^{55}, \\
& K_{y z}^{l j \sigma \tau}=I_{l j}\left(J_{\sigma, y, z}^{23}+J_{\sigma, z}^{44, y}\right), \\
& K_{z y}^{l j \sigma \tau}=I_{l j}\left(J_{\sigma, y}^{44, z}+J_{\sigma, z}^{23} \tau, y\right) .
\end{aligned}
$$

The generic term $J_{\sigma_{(, \eta)}}^{g h} \tau_{(, \xi)}$ is a cross-section moment:

$$
J_{\sigma_{(, \eta)} \tau_{(, \xi)}}^{g h}=C_{g h} \int_{\Omega_{e}} F_{\sigma_{(, \eta)}} F_{\tau_{(, \xi)}} d \Omega \quad \text { with }(\eta, \xi)=\{y, z\}^{2}
$$

and it is a weighted sum (in the continuum) of each elemental cross-section area where the weight functions account for the spatial distribution of the geometry and the material. $I_{l_{(, x)} j_{(, x)}}$ is an integral along the element axis of the product of the shape functions and/or their derivatives:

$$
I_{l_{(, x)} j_{(, x)}}=\int_{l_{e}} N_{l_{(, x)}} N_{j_{(, x)}} d x .
$$

These integrals are evaluated numerically through Gauss' quadrature method. In order to correct the shear locking, a selective integration technique is used. The selected under-integrated term is $I_{l j}$ in $K_{x x}^{l j \sigma \tau}$ that is related to shear deformations $\gamma_{x y}$ and $\gamma_{x z}$.

\subsection{Virtual variation of the external work}

The beam can be loaded by concentrated forces $(\mathbf{F})$, surface loads (p) and line loads (l). The virtual variation of the external work reads:

$$
\delta \mathscr{L}_{\mathrm{ext}}^{e}=\delta \mathscr{L}_{\mathrm{ext}}^{e F}+\delta \mathscr{L}_{\mathrm{ext}}^{e p}+\delta \mathscr{L}_{\mathrm{ext}}^{e l} .
$$

\subsubsection{Force loads}

The virtual work of a generic force load:

$$
\mathbf{F}^{T}=\left\{\begin{array}{lll}
F_{x} & F_{y} & F_{z}
\end{array}\right\},
$$

applied on a generic point $\mathbf{x}_{F}=\left(x_{F}, y_{F}, z_{F}\right)$ is:

$$
\delta \mathscr{L}_{\text {ext }}^{e F}=\delta \mathbf{u}^{T}\left(\mathbf{x}_{F}\right) \mathbf{F} .
$$

By replacing Eq. (19) computed at $\mathbf{x}_{F}$ within Eq. (32), the virtual work of a point force becomes:

$$
\delta \mathscr{L}_{\mathrm{ext}}^{e F}=\delta \mathbf{q}_{\mathcal{D}_{\Omega} \sigma}^{s T} \mathbf{F}_{\mathcal{D}_{x} \sigma}^{s}+\delta \mathbf{q}_{\mathcal{D}_{x} l}^{s T} \mathbf{F}_{\mathcal{D}_{\Omega} l}^{s},
$$

where $\mathbf{F}_{\mathcal{D}_{x} \sigma}^{s}$ and $\mathbf{F}_{\mathcal{D}_{\Omega} l}^{s} \in \mathbb{R}^{3}$ are the force load vectors projected over $\mathcal{D}_{x}$ and $\mathcal{D}_{\Omega}$, respectively. They are variationally consistent with the kinematic approximation 
over the cross-section and the finite element approximation over the beam axis:

$$
\begin{aligned}
& \mathbf{F}_{\mathcal{D}_{x} \sigma}^{s}=\mathbf{q}_{\mathcal{D}_{x} l}^{s} \circ \mathbf{F}_{\sigma l}\left(\mathbf{x}_{F}\right)=\mathbf{q}_{\mathcal{D}_{x} l}^{s} \circ F_{\sigma}\left(y_{F}, z_{F}\right) N_{l}\left(x_{F}\right) \mathbf{F}, \\
& \mathbf{F}_{\mathcal{D}_{\Omega} l}^{s}=\mathbf{q}_{\mathcal{D}_{\Omega} \sigma}^{s} \circ \mathbf{F}_{\sigma l}\left(\mathbf{x}_{F}\right)=\mathbf{q}_{\mathcal{D}_{\Omega} \sigma}^{s} \circ F_{\sigma}\left(y_{F}, z_{F}\right) N_{l}\left(x_{F}\right) \mathbf{F} .
\end{aligned}
$$

The term $\mathbf{F}_{\sigma l} \in \mathbb{R}^{3}$, defined as:

$$
\mathbf{F}_{\sigma l}=F_{\sigma}\left(y_{F}, z_{F}\right) N_{l}\left(x_{F}\right) \mathbf{F}
$$

is the fundamental nucleus of the force load vector as derived from the classical finite element formulation.

\subsubsection{Surface loads}

The following surface loads $\mathbf{p}_{y}(x, z)$ and $\mathbf{p}_{z}(x, y)$ are considered:

$$
\begin{aligned}
& \mathbf{p}_{y}^{T}(x, z)=\left\{\begin{array}{lll}
p_{y x} & p_{y y} & \left.p_{y z}\right\}
\end{array}\right\}(y, z) \in\left\{y=y_{p_{y}}, z \in\left[z_{p_{y} 1}, z_{p_{y} 2}\right]\right\}, \\
& \mathbf{p}_{z}^{T}(x, y)=\left\{\begin{array}{lll}
p_{z x} & p_{z y} & \left.p_{z z}\right\}
\end{array}\right\}(y, z) \in\left\{y \in\left[y_{p_{z} 1}, y_{p_{z} 2}\right], z=z_{p_{z}}\right\} .
\end{aligned}
$$

They act on surfaces whose normal is parallel to the $y$ - or $z$-axis and along the whole axial span of the element. The first subscript accounts for the normal of the surface the load is applied upon, whereas the second one stands for the load direction of application. The virtual variation of the external work is:

$$
\begin{aligned}
\delta \mathscr{L}_{\mathrm{ext}}^{e p}= & \int_{l_{e}} \int_{z_{p_{y} 1}}^{z_{p_{y} 2}} \delta \mathbf{u}^{T}\left(x, y_{p_{y}}, z\right) \mathbf{p}_{y}(x, z) d z d x \\
& +\int_{l_{e}} \int_{y_{p_{z} 1}}^{y_{p_{z} 2}} \delta \mathbf{u}^{T}\left(x, y, z_{p_{z}}\right) \mathbf{p}_{z}(x, y) d y d x .
\end{aligned}
$$

Within the proposed framework, the virtual work of the surface loads is:

$$
\delta \mathscr{L}_{\mathrm{ext}}^{e p}=\delta \mathbf{q}_{\mathcal{D}_{\Omega} \sigma}^{s T}\left(\mathbf{P}_{z \mathcal{D}_{x} \sigma}^{s}+\mathbf{P}_{y \mathcal{D}_{x} \sigma}^{s}\right)+\delta \mathbf{q}_{\mathcal{D}_{x} l}^{s T}\left(\mathbf{P}_{z \mathcal{D}_{\Omega} l}^{s}+\mathbf{P}_{y \mathcal{D}_{\Omega} l}^{s}\right),
$$

being:

$$
\begin{aligned}
\left(\mathbf{P}_{z \mathcal{D}_{x} \sigma}^{s}, \mathbf{P}_{y \mathcal{D}_{x} \sigma}^{s}\right) & =\mathbf{q}_{\mathcal{D}_{x} l}^{s} \circ\left(\mathbf{P}_{z \sigma l}, \mathbf{P}_{y \sigma l}\right), \\
\left(\mathbf{P}_{z \mathcal{D}_{\Omega} l}^{s}, \mathbf{P}_{y \mathcal{D}_{\Omega} l}^{s}\right) & =\mathbf{q}_{\mathcal{D}_{\Omega} \sigma}^{s} \circ\left(\mathbf{P}_{z \sigma l}, \mathbf{P}_{y \sigma l}\right),
\end{aligned}
$$

where:

$$
\begin{aligned}
\mathbf{P}_{z \sigma l} & =\int_{l_{e}} N_{l}(x) \int_{y_{p_{z} 1}}^{y_{p_{z} 2}} F_{\sigma}\left(y, z_{p_{z}}\right) \mathbf{p}_{z}(x, y) d y d x, \\
\mathbf{P}_{y \sigma l} & =\int_{l_{e}} N_{l}(x) \int_{z_{p_{y} 1}}^{z_{p_{y} 2}} F_{\sigma}\left(y_{p_{y}}, z\right) \mathbf{p}_{y}(x, z) d z d x,
\end{aligned}
$$

are the classical finite element fundamental nuclei of the surface loads. 


\subsubsection{Line loads}

A line load acting on a straight line parallel to the beam axis and spanning along the whole element length is considered:

$$
\mathbf{l}^{T}(x)=\left\{\begin{array}{lll}
l_{x} & l_{y} & l_{z}
\end{array}\right\}
$$

The position of the load over the cross-section is $\left(y_{l}, z_{l}\right)$. The virtual external work is:

$$
\delta \mathscr{L}_{\text {ext }}^{e l}=\int_{l_{e}} \delta \mathbf{u}^{T}\left(x, y_{l}, z_{l}\right) \mathbf{l}(x) d x
$$

and its form variationally consistent with the considered beam models and PGD framework is:

$$
\delta \mathscr{L}_{\mathrm{ext}}^{e l}=\delta \mathbf{q}_{\mathcal{D}_{\Omega} \sigma}^{s T} \mathbf{L}_{\mathcal{D}_{x} \sigma}^{s}+\delta \mathbf{q}_{\mathcal{D}_{x} l}^{s T} \mathbf{L}_{\mathcal{D}_{\Omega} l}^{s}
$$

where:

$$
\begin{aligned}
\left(\mathbf{L}_{\mathcal{D}_{x} \sigma}^{s}, \mathbf{L}_{\mathcal{D}_{\Omega} l}^{s}\right) & =\left(\mathbf{q}_{\mathcal{D}_{x} l}^{s}, \mathbf{q}_{\mathcal{D}_{\Omega} \sigma}^{s}\right) \circ \mathbf{L}_{\sigma l}\left(y_{l}, z_{l}\right), \\
\mathbf{L}_{\sigma l}\left(y_{l}, z_{l}\right) & =F_{\sigma}\left(y_{l}, z_{l}\right) \int_{l_{e}} N_{l}(x) \mathbf{l}(x) d x
\end{aligned}
$$

is the finite element fundamental nucleus of the load vector.

For the sake of conciseness, the following generic notation for the external loads is introduced:

$$
\begin{aligned}
& \mathbf{R}_{\mathcal{D}_{x} \sigma}^{s}=\mathbf{F}_{\mathcal{D}_{x} \sigma}^{s}+\mathbf{P}_{z \mathcal{D}_{x} \sigma}^{s}+\mathbf{P}_{y \mathcal{D}_{x} \sigma}^{s}+\mathbf{L}_{\mathcal{D}_{x} \sigma}^{s}=\mathbf{q}_{\mathcal{D}_{x} l}^{s} \circ \mathbf{R}_{\sigma l} \\
& \mathbf{R}_{\mathcal{D}_{\Omega} l}^{s}=\mathbf{F}_{\mathcal{D}_{\Omega} l}^{s}+\mathbf{P}_{z \mathcal{D}_{\Omega} l}^{s}+\mathbf{P}_{y \mathcal{D}_{\Omega} l}^{s}+\mathbf{L}_{\mathcal{D}_{\Omega} l}^{s}=\mathbf{q}_{\mathcal{D}_{\Omega} \sigma}^{s} \circ \mathbf{R}_{\sigma l},
\end{aligned}
$$

with:

$$
\mathbf{R}_{\sigma l}=\mathbf{F}_{\sigma l}+\mathbf{P}_{z \sigma l}+\mathbf{P}_{y \sigma l}+\mathbf{L}_{\sigma l}
$$

\section{Problem Formulation and Solution}

\subsection{Assembling procedure at element level}

Two coupled nonlinear equations are obtained by replacing the expressions of the virtual strain energy and the external works within the PVD statement:

$$
\begin{gathered}
\delta \mathbf{q}_{\mathcal{D}_{\Omega} \sigma}^{s}: \mathbf{K}_{\mathcal{D}_{x}}^{\sigma \tau s i} \mathbf{q}_{\mathcal{D}_{\Omega} \tau}^{i}=\mathbf{R}_{\mathcal{D}_{x} \sigma}^{s}, \\
\delta \mathbf{q}_{\mathcal{D}_{x} l}^{s}: \mathbf{K}_{\mathcal{D}_{\Omega}}^{l j s i} \mathbf{q}_{\mathcal{D}_{x} j}^{i}=\mathbf{R}_{\mathcal{D}_{\Omega} l}^{s} .
\end{gathered}
$$

Accounting for Eqs. (25) and (46), Eqs. (48) read:

$$
\begin{gathered}
\left(\mathbf{Q}_{\mathcal{D}_{x} l}^{s} \mathbf{K}^{l j \sigma \tau} \mathbf{Q}_{\mathcal{D}_{x} j}^{i}\right) \mathbf{q}_{\mathcal{D}_{\Omega} \tau}^{i}=\mathbf{q}_{\mathcal{D}_{x} l}^{s} \circ \mathbf{R}_{\sigma l}, \\
\left(\mathbf{Q}_{\mathcal{D}_{\Omega} \sigma}^{s} \mathbf{K}^{l j \sigma \tau} \mathbf{Q}_{\mathcal{D}_{\Omega} \tau}^{i}\right) \mathbf{q}_{\mathcal{D}_{x} j}^{i}=\mathbf{q}_{\mathcal{D}_{\Omega} \sigma}^{s} \circ \mathbf{R}_{\sigma l} .
\end{gathered}
$$

Once the expansion order over the cross-section $N$ and the number of nodes per element $N_{n}$ are fixed, the nucleus of the problem over the cross-section has to be 
expanded over the indexes $\sigma$ and $\tau$ in $\left\{1,2, \ldots, N_{u}\right\}$ and summed over the element nodes indexes $l$ and $j$ in $\left\{1,2, \ldots, N_{n}\right\}$ :

$$
\begin{gathered}
\mathbf{q}_{\mathcal{D}_{\Omega} \tau}^{i} \in \mathbb{R}^{3} \rightarrow \mathbf{q}_{\mathcal{D}_{\Omega}}^{i} \in \mathbb{R}^{3 N_{u}}, \\
\mathbf{R}_{\mathcal{D}_{x} \sigma}^{s} \in \mathbb{R}^{3} \rightarrow \mathbf{R}_{\mathcal{D}_{x}}^{s} \in \mathbb{R}^{3 N_{u}}, \\
\mathbf{K}_{\mathcal{D}_{x} \sigma s i}^{\sigma \tau s i} \in \mathbb{R}^{3 \times 3} \rightarrow \mathbf{K}_{\mathcal{D}_{x}}^{s i} \in \mathbb{R}^{3 N_{u} \times 3 N_{u}} .
\end{gathered}
$$

The expansion procedure increases the dimension (from $\mathbb{R}^{3}$ to $\mathbb{R}^{3 N_{u}}$ ) of the expanded quantity: its nuclear form is "unfolded" over the whole approximation space. Expansion and summation are graphically presented in Fig. 2. The dimension of the problem $\left(N_{\mathcal{D}_{\Omega}}\right)$ to be solved over the cross-section domain is:

$$
N_{\mathcal{D}_{\Omega}}=3 N_{u}=3 \frac{(N+1)(N+2)}{2}
$$

The nucleus of the companion problem over the beam axis has to be expanded over the indexes $l$ and $j$ and summed over the cross-section indexes $\sigma$ and $\tau$, see

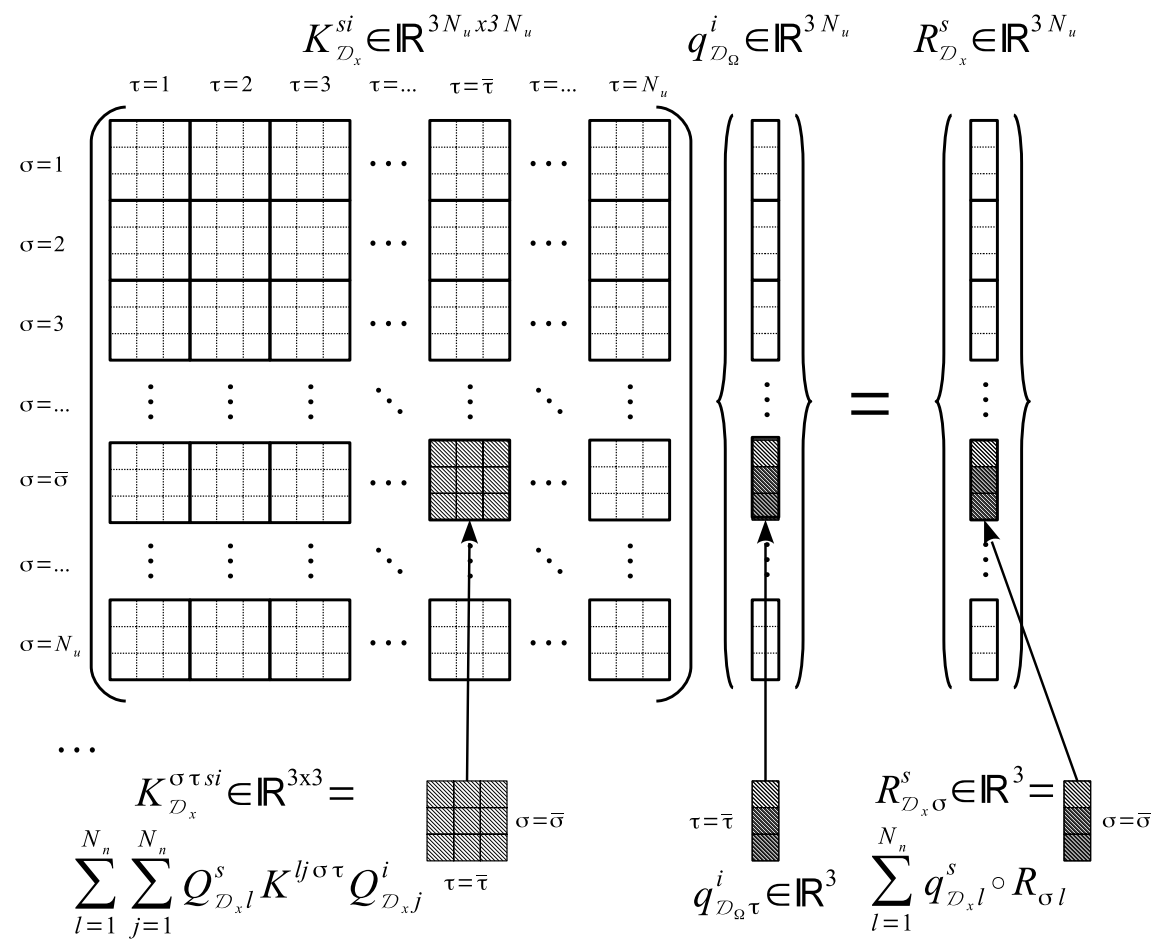

Fig. 2. Assembling procedure of the cross-section problem $\mathcal{D}_{\Omega}$ at element level. 


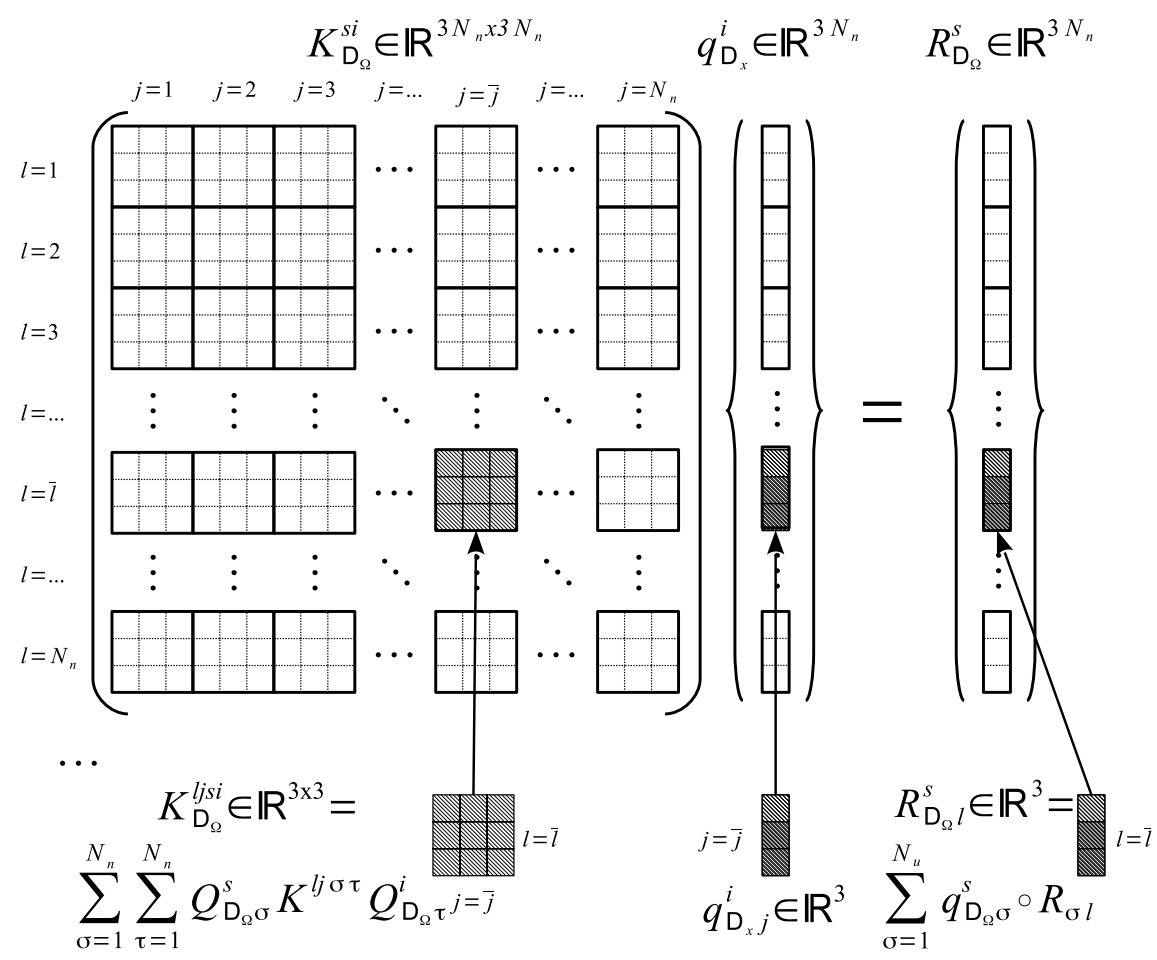

Fig. 3. Assembling procedure of the axial finite element problem $\mathcal{D}_{x}$ at element level.

Fig. 3:

$$
\begin{aligned}
& \mathbf{q}_{\mathcal{D}_{x} j}^{i} \in \mathbb{R}^{3} \rightarrow \mathbf{q}_{\mathcal{D}_{x}}^{i} \in \mathbb{R}^{3 N_{n}}, \\
& \mathbf{R}_{\mathcal{D}_{\Omega} l}^{s} \in \mathbb{R}^{3} \rightarrow \mathbf{R}_{\mathcal{D}_{\Omega}}^{s} \in \mathbb{R}^{3 N_{n}}, \\
& \mathbf{K}_{\mathcal{D}_{\Omega}}^{l j s i} \in \mathbb{R}^{3 \times 3} \rightarrow \mathbf{K}_{\mathcal{D}_{\Omega}}^{s i} \in \mathbb{R}^{3 N_{n} \times 3 N_{n}} .
\end{aligned}
$$

After this assembling procedure, the problems in Eqs. (48) become:

$$
\begin{aligned}
& \mathbf{K}_{\mathcal{D}_{x}}^{s i} \mathbf{q}_{\mathcal{D}_{\Omega}}^{i}=\mathbf{R}_{\mathcal{D}_{x}}^{s}, \\
& \mathbf{K}_{\mathcal{D}_{\Omega}}^{s i} \mathbf{q}_{\mathcal{D}_{x}}^{i}=\mathbf{R}_{\mathcal{D}_{\Omega}}^{s}
\end{aligned}
$$

and hold at element level.

\subsection{Assembling procedure at structural level}

The problem at structural level is obtained by writing the PVD statement for the whole beam:

$$
\delta \mathscr{L}_{\text {int }}-\delta \mathscr{L}_{\text {ext }}=\sum_{e=1}^{N_{e}}\left(\delta \mathscr{L}_{\text {int }}^{e}-\delta \mathscr{L}_{\text {ext }}^{e}\right)=0
$$


where $N_{e}$ is the number of finite elements used to discretize the beam axis. The dimension of the problem to be solved over the axial domain is:

$$
N_{\mathcal{D}_{x}}=3\left[N_{e}\left(N_{n}-1\right)+1\right] .
$$

It is worth underlining that the assembling procedure at structural level derives from the finite element solution method or, more generally, from a weak form solution type. Therefore, it directly affects the problem on the beam axis domain $\mathcal{D}_{x}$ only. The unknown vector $\mathbf{q}_{\mathcal{D}_{x}}^{i}$, the stiffness matrix $\mathbf{K}_{\mathcal{D}_{\Omega}}^{s i}$ and the load vector $\mathbf{F}_{\mathcal{D}_{\Omega}}^{s}$ are expanded over the discretization domain using classical finite element assembling procedures based upon the congruency and equilibrium at a node shared by two consecutive elements [Bathe, 1996]:

$$
\begin{aligned}
\mathbf{q}_{\mathcal{D}_{x}}^{i} \in \mathbb{R}^{3 N_{n}} & \rightarrow \hat{\mathbf{q}}_{\mathcal{D}_{x}}^{i} \in \mathbb{R}^{N_{\mathcal{D}_{x}}}, \\
\mathbf{R}_{\mathcal{D}_{\Omega}}^{s} \in \mathbb{R}^{3 N_{n}} & \rightarrow \hat{\mathbf{R}}_{\mathcal{D}_{\Omega}}^{s} \in \mathbb{R}^{N_{\mathcal{D}_{x}}}, \\
\mathbf{K}_{\mathcal{D}_{\Omega}}^{s i} \in \mathbb{R}^{3 N_{n} \times 3 N_{n}} & \rightarrow \hat{\mathbf{K}}_{\mathcal{D}_{\Omega}}^{s i} \in \mathbb{R}^{N_{\mathcal{D}_{x}} \times N_{\mathcal{D}_{x}}} .
\end{aligned}
$$

The problem on $\mathcal{D}_{\Omega}$ is affected indirectly by this further assembling step because of its coupling with the problem on $\mathcal{D}_{x}$. The dimension of this latter problem, therefore, does not change and it is equal to $N_{\mathcal{D}_{\Omega}}$. The stiffness matrix $\mathbf{K}_{\mathcal{D}_{x}}^{\text {si }}$ and the load vector $\mathbf{R}_{\mathcal{D}_{x}}^{s}$ of each element are simply summed. Each element contributes to the problem on the cross-section in an equivalent single layer sense:

$$
\begin{gathered}
\mathbf{q}_{\mathcal{D}_{\Omega}}^{i} \in \mathbb{R}^{N_{\mathcal{D}_{\Omega}}} \rightarrow \hat{\mathbf{q}}_{\mathcal{D}_{\Omega}}^{i} \in \mathbb{R}^{N_{\mathcal{D}_{\Omega}}}, \\
\mathbf{R}_{\mathcal{D}_{x}}^{s} \in \mathbb{R}^{N_{\mathcal{D}_{\Omega}}} \rightarrow \hat{\mathbf{R}}_{\mathcal{D}_{x}}^{s} \in \mathbb{R}^{N_{\mathcal{D}_{\Omega}}}, \\
\mathbf{K}_{\mathcal{D}_{x}}^{s i} \in \mathbb{R}^{N_{\mathcal{D}_{\Omega}} \times N_{\mathcal{D}_{\Omega}}} \rightarrow \hat{\mathbf{K}}_{\mathcal{D}_{x}}^{s i} \in \mathbb{R}^{N_{\mathcal{D}_{\Omega}} \times N_{\mathcal{D}_{\Omega}}} .
\end{gathered}
$$

Finally, the problem for the whole beam within a PGD framework reads:

$$
\begin{aligned}
\hat{\mathbf{K}}_{\mathcal{D}_{x}}^{s i} \hat{\mathbf{q}}_{\mathcal{D}_{\Omega}}^{i} & =\hat{\mathbf{R}}_{\mathcal{D}_{x}}^{s}, \\
\hat{\mathbf{K}}_{\mathcal{D}_{\Omega}}^{s i} \hat{\mathbf{q}}_{\mathcal{D}_{x}}^{i} & =\hat{\mathbf{R}}_{\mathcal{D}_{\Omega}}^{s} .
\end{aligned}
$$

\subsection{Solution over the number of couples}

As final step, the global problem in Eqs. (58) needs to be solved for each couple. The solution procedure proposed by Ammar et al. [2006] is here used. On this regard, the indexes $s$ and $i$ ranging over the number of couples $N_{c}$ play a different role than indexes $(l, j)$ and $(\sigma, \tau)$ do. These latter derive from the UF and are used to expand the nucleus of the stiffness matrices and load vectors to obtain the corresponding global terms. The former derive from the PGD. Matrices and vectors in Eqs. (58) are not expanded over $s$ and $i$. A summation over the index $i \in\{1,2, \ldots, s\}$ is performed instead and the dimensions of the two problems over $\mathcal{D}_{\Omega}$ and $\mathcal{D}_{x}$ do not change. 
In the case of an unknown couple $s$ and supposing that $\hat{\mathbf{q}}_{\mathcal{D}_{\Omega}}^{r}$ and $\hat{\mathbf{q}}_{\mathcal{D}_{x}}^{r}$ with $r \in\{1,2, \ldots, s-1\}$ have been already computed, Eqs. (58) become:

$$
\begin{aligned}
\hat{\mathbf{K}}_{\mathcal{D}_{x}}^{s s} \hat{\mathbf{q}}_{\mathcal{D}_{\Omega}}^{s} & =\hat{\mathbf{R}}_{\mathcal{D}_{x}}^{s}-\hat{\mathbf{K}}_{\mathcal{D}_{x}}^{s r} \hat{\mathbf{q}}_{\mathcal{D}_{\Omega}}^{r}, \\
\hat{\mathbf{K}}_{\mathcal{D}_{\Omega}}^{s s} \hat{\mathbf{q}}_{\mathcal{D}_{x}}^{s} & =\hat{\mathbf{R}}_{\mathcal{D}_{\Omega}}^{s}-\hat{\mathbf{K}}_{\mathcal{D}_{\Omega}}^{s r} \hat{\mathbf{q}}_{\mathcal{D}_{x}}^{r} .
\end{aligned}
$$

The second term in each right-hand side of the previous equations is summed over $r \in\{1,2, \ldots, s-1\}$ and it represents a residual term. The solution of Eqs. (59) is obtained through a row-wise scheme: the residuals account for the interaction between the couple currently computed with those already determined. In this sense, each new couple represents a solution refinement. The matrices $\hat{\mathbf{K}}_{\mathcal{D}_{x}}^{s r}$ and $\hat{\mathbf{K}}_{\mathcal{D}_{\Omega}}^{s r}$ are not, in general, symmetric because they are computed using the unknown vectors of different couples $(r \neq s)$. As far as the left hand side terms of Eqs. (59) are concerned, Einstein's notation does not apply for $s$ since it is repeated more than twice. A fixed point method is, then, used to solve the coupled nonlinear problem in Eqs. (59):

(i) a tentative solution $\hat{\mathbf{q}}_{\mathcal{D}_{x}}^{s_{0}}$ is first set for the problem on $\mathcal{D}_{\Omega}$ and $\hat{\mathbf{q}}_{\mathcal{D}_{\Omega}}^{s_{0}}$ is obtained,

(ii) $\hat{\mathbf{q}}_{\mathcal{D}_{\Omega}}^{s_{0}}$ is, then, used for the problem on $\mathcal{D}_{x}$ and $\hat{\mathbf{q}}_{\mathcal{D}_{x}}^{s_{1}}$ is computed,

(iii) the procedure at the previous two points is iterated until the following convergence criterion is satisfied:

$$
\max \left(\frac{\left\|\hat{q}_{\mathcal{D}_{x}}^{s_{m}}-\hat{q}_{\mathcal{D}_{x}}^{s_{m-1}}\right\|_{2}}{\left\|\hat{q}_{\mathcal{D}_{x}}^{s_{m-1}}\right\|_{2}}, \frac{\left\|\hat{q}_{\mathcal{D}_{\Omega}}^{s_{m}}-\hat{q}_{\mathcal{D}_{\Omega}}^{s_{m-1}}\right\|_{2}}{\left\|\hat{q}_{\mathcal{D}_{\Omega}}^{s_{m-1}}\right\|_{2}}\right) \leq \varepsilon
$$

being $\varepsilon=10^{-6}$ a convergence tolerance and $\|\square\|_{2}$ the Euclidean norm.

For the considered numerical investigation, it has been observed that the number of iteration required to reach the convergence increases for each successive couple. Nevertheless, about 15 iterations have been required, at worst. At each iteration, two problems of dimension $N_{\mathcal{D}_{\Omega}}$ and $N_{\mathcal{D}_{x}}$ are solved. It should be noticed that the order of an equivalent classical finite element problem is $3 \cdot \frac{(N+1)(N+2)}{2} \cdot\left[N_{e}\left(N_{n}-1\right)+1\right]$ or, equivalently, $\frac{N_{\mathcal{D}_{\Omega}} \cdot N_{\mathcal{D}_{x}}}{3}$.

\subsection{Boundary conditions}

As far as Dirichlet boundary conditions are concerned, the case of nil prescribed displacements along the beam axis is considered. The same principle as for the assembling procedure at structural level is valid. A displacements' constrain is directly imposed for the problem on the axial space $\mathcal{D}_{x}$ :

$$
u_{\varphi}\left(x_{\underline{j}}, y, z\right)=F_{\tau}(y, z) N_{\underline{j}}\left(x_{\underline{j}}\right) q_{\varphi \mathcal{D}_{\Omega} \tau}^{i} q_{\varphi \mathcal{D}_{x} \underline{j}}^{i}=0,
$$

where subscript $\varphi$ stands for a generic spatial coordinate, $\underline{j}$ for the constrained node index and $x_{\underline{j}}$ for its axial position. Summation over the reaped index $\underline{j}$ is not 
actually performed because of the well known properties of the shape functions and Eq. (61) reduces to:

$$
q_{\varphi \mathcal{D}_{x} \underline{j}}^{i}=0 .
$$

A numerical penalization technique is used for imposing a nil displacement: the diagonal term of the stiffness matrix $\hat{\mathbf{K}}_{\mathcal{D}_{\Omega}}^{\text {si }}$ corresponding to the constrained degree of freedom is set to a value two or three order of magnitude higher than the maximum absolute value of the matrix [Bathe, 1996].

For the problem on the cross-section domain, the boundary conditions are imposed indirectly during the projection of the stiffness matrix. According to Eqs. (25) and (57), a constrained degree of freedom does not yield any contribution to the assembling procedure over the whole structure being its value equal to zero.

\section{Numerical Results and Discussion}

Beams are made of an aluminium alloy whose properties are: Young's modulus equal to $72 \mathrm{GPa}$ and Poisson's ratio equal to 0.33 .

The maximal length of the sides of the cross-section $a$ and $b$ are equal to $1 \mathrm{~m}$. A length-to-side ratio as high as 100 (slender beams) and as low as five (short beams) is used.

Simply supported and clamped-clamped beams are considered. In the case of simply supported boundary conditions, results are compared with a closed form, Navier-type method [Giunta et al., 2011] where uniform loads along the beam axis are approximated by means of their Fourier series expansion. A convergence of displacements and stresses up to four significant digits is obtained by considering the highest harmonic term in the Fourier series expansion having 200 half-waves. It should be noted that the Navier-type results are exact within the theory approximation. Surface or line loads are uniformly distributed along the whole beam length. The former load is adopted to investigate beams under bending, torsion is studied through the latter.

Three-dimensional finite element results obtained via the commercial code ANSYS are also presented. The three-dimensional 20-node quadratic element "Solid186" is used. For each analysis, a coarse and a fine mesh are considered in order to address the convergence of the three-dimensional finite element solution. These solutions are addressed as FEM 3D-C and FEM 3D-R, respectively.

\subsection{Square cross-section beams under bending}

A surface load $p_{y y}$ equal to $1 \mathrm{~Pa}$ is applied as shown in Fig. 4. Simply supported boundary conditions are first considered.

A convergence analysis versus the number of elements and the number of couples is presented in Fig. 5 in the case of short beams. A second-order model is used. Results for slender beams and a cross-section approximation order higher than two 


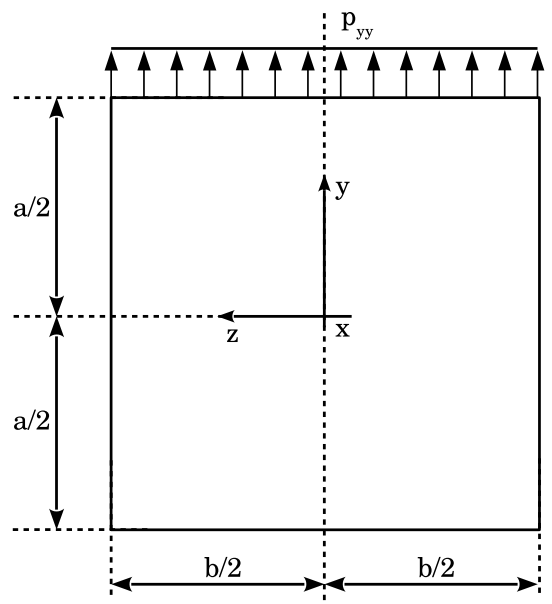

Fig. 4. Square cross-section geometry and surface bending load.

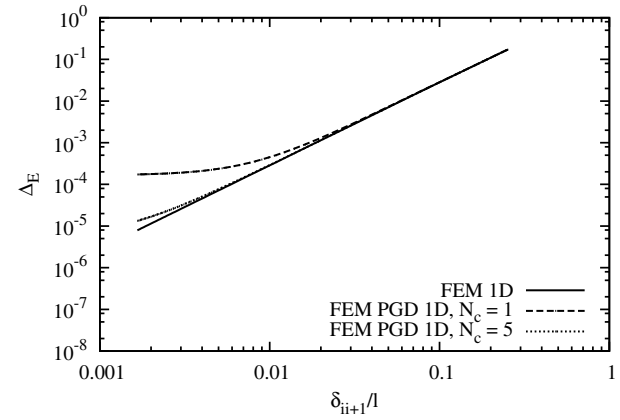

(a)

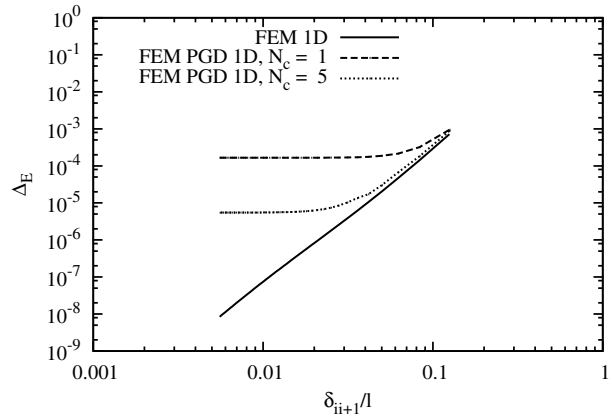

(b)

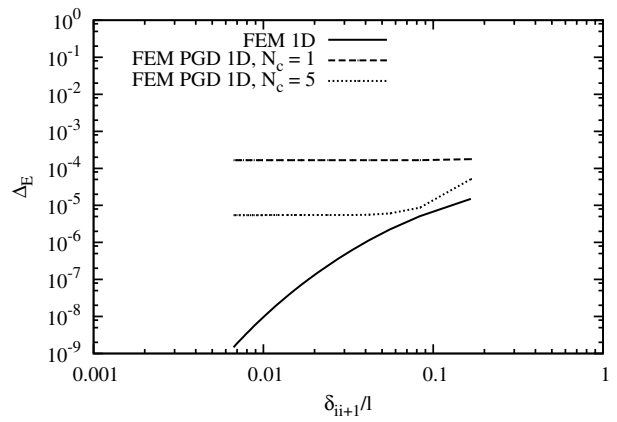

(c)

Fig. 5. Strain energy relative error $\Delta_{E}$ versus the normalized distance $\delta_{i i+1} / l$ between two consecutive nodes for (a) linear, (b) quadratic and (c) cubic element. Simply supported beam, $l / a=10$ and $N=2$. 
are very similar and they are not reported for the sake of brevity. The normalized difference in strain energy $\mathscr{L}_{\text {int }}$ is defined as:

$$
\Delta_{E}=\frac{\mathscr{L}_{\text {int }}^{\mathrm{Nav}}-\mathscr{L}_{\mathrm{int}}}{\mathscr{L}_{\text {int }}^{\mathrm{Nav}}},
$$

where superscript "Nav" stands for the Navier-type solution. $\Delta_{E}$ is plotted versus the distance between two consecutive nodes $\delta_{i i+1}$ normalized versus the beam length $l$. This parameter has been introduced in order to compare the convergence between linear, quadratic and cubic elements for the same number of nodes. The classical one-dimensional finite element solution (FEM 1D) is also presented for the sake of comparison. For the classical finite element solution, the error in the strain energy decreases as the number of nodes increases and, for a fixed number of nodes, the higher the order of the finite element shape functions, the smaller the error. As far as the PGD is concerned, the error presents an horizontal asymptote. The order of magnitude of the error is $10^{-3}$ and $10^{-6}$ for $N_{c}$ equal to one and five regardless the element type. Unless differently stated, the results further presented are computed using 121 nodes that corresponds to 120 B2 elements, 60 B3 ones and 40 B4 elements.

The effect of the number of couples on the convergence of the solution in terms of strain energy is presented in Fig. 6. Classical FEM and FEM PGD solutions using linear and cubic elements are shown. Results are for a short beam and second-order models. As the number of couples increases, the PGD solution converges to the corresponding FEM one. In the remaining of the numerical investigations, $N_{c}$ equal to six and seven is used for $l / a=100$ and 10, respectively.

As far as shear locking is concerned, Fig. 7 presents the variation of the ratio $\tilde{u}_{y}$ defined as:

$$
\tilde{u}_{y}=\frac{u_{y}\left(\frac{l}{2}, 0,0\right)}{u_{y}^{\operatorname{Nav}}\left(\frac{l}{2}, 0,0\right)}
$$

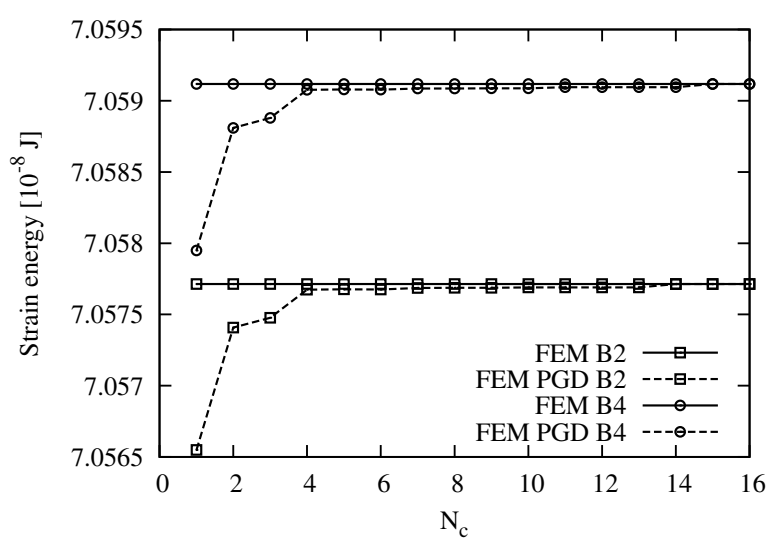

Fig. 6. Strain energy convergence versus the number of couples, B2 and B4 elements, $l / a=10$ and $N=2$. 


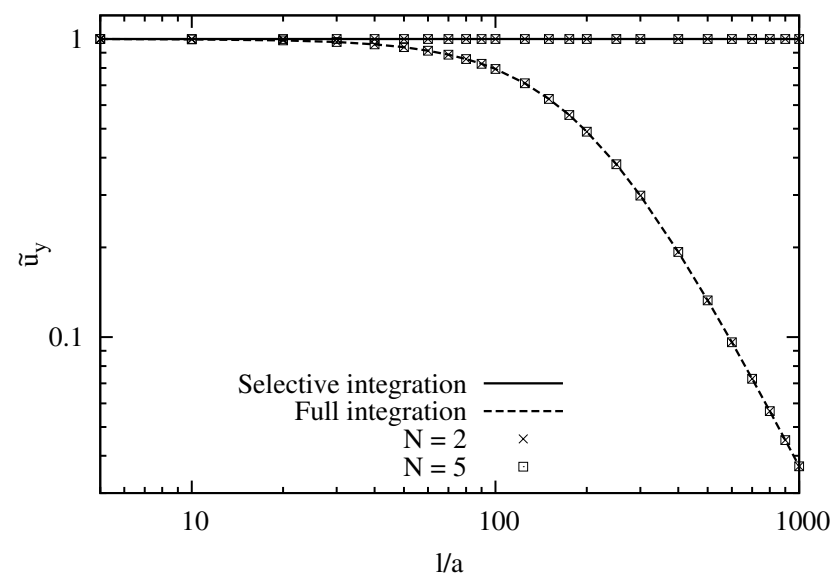

Fig. 7. Transverse displacement ratio $\tilde{u}_{y}=u_{y}(l / 2,0,0) / u_{y}^{\mathrm{Nav}}(l / 2,0,0)$ versus $l / a$ via linear elements, $N=2$ and 5 . The figure is the same for the FEM as well as FEM PGD solution regardless the value of $N_{c}$.

versus the length-to-side ratio $l / a$. Very short $(l / a=5)$ up to very slender $(l / a=$ 1000) beams are considered. Results have been computed using second- and fifthorder theories. Both full and selective integration have been used. Only B2 elements are presented because they are the most affected by shear locking (in the case of B3 elements, results between selective and full integration for $l / a=1000$ differ by about $0.05 \%$ only). The figure clearly shows that the solution obtained by full integration locks as soon as the beam becomes slender $(l / a \geq 30)$, whereas a selective integration yields locking free results. The correction of the locking is effective regardless the approximation order over the cross-section since the results for $N$ equal to two and five are coincident. Furthermore, results are the same regardless the manner they have been obtained (either by the classical finite element method or within a PGD framework). The reason is that shear locking is a numerical phenomenon due to the finite element approximation only.

Tables 2-4 present the following nondimensionalized displacements:

$$
\left(\bar{u}_{x}, \bar{u}_{y}, \bar{u}_{z}\right)=\frac{4 E a}{l^{2} p_{y y}}\left(u_{x}, u_{y}, u_{z}\right),
$$

where $\bar{u}_{x}$ is evaluated at $(0,-a / 2,+b / 2), \bar{u}_{y}$ at $(l / 2,+a / 2,0)$ and $\bar{u}_{z}$ at $(l / 2,+a / 2,+b / 2)$. Unless differently stated, for each set of tabular results, reference three-dimensional finite element and Navier-type solution (whenever applicable) are presented first as in Table 2 where the displacements for slender and short beams are shown. These results provide insight about the accuracy of the proposed one-dimensional beam models. A sixth-order theory matches the refined three-dimensional FEM solution up to four significant digits. Tables 3 and 4 compare the one-dimensional beam FEM and FEM PGD results. In this manner, the effect of the classical finite element approximation and of the PGD on the accuracy 
Table 2. Dimensionless displacements for slender and short simply supported beams under a surface bending load via three-dimensional finite element and Navier-type solutions.

\begin{tabular}{|c|c|c|c|c|c|c|}
\hline & \multicolumn{3}{|c|}{$l / a=100$} & \multicolumn{3}{|c|}{$l / a=10$} \\
\hline & $10^{-2} \times \bar{u}_{x}$ & $10^{-3} \times \bar{u}_{y}$ & $-10 \times \bar{u}_{z}$ & $10^{-1} \times \bar{u}_{x}$ & $10^{-1} \times \bar{u}_{y}$ & $-10 \times \bar{u}_{z}$ \\
\hline FEM 3D-R ${ }^{a}$ & 1.000 & 6.251 & 4.951 & 1.002 & 6.362 & 5.018 \\
\hline FEM 3D-C ${ }^{b}$ & 1.000 & 6.251 & 4.951 & 1.002 & 6.362 & 5.019 \\
\hline$N=6$ & 1.000 & 6.251 & 4.951 & 1.002 & 6.362 & 5.018 \\
\hline$N=5$ & 1.000 & 6.251 & 4.951 & 1.002 & 6.362 & 5.017 \\
\hline$N=4$ & 1.000 & 6.251 & 4.951 & 1.001 & 6.362 & 5.016 \\
\hline$N=3$ & 1.000 & 6.251 & 4.951 & 1.001 & 6.362 & 5.014 \\
\hline$N=2$ & 1.000 & 6.251 & 4.951 & 0.999 & 6.340 & 5.002 \\
\hline
\end{tabular}

${ }^{a}$ Refined mesh: $40 \times 40 \times 400$ for $l / a=100$ and $80 \times 80 \times 80$ for $l / a=10$.

${ }^{\mathrm{b}}$ Coarse mesh: $10 \times 10 \times 100$ for $l / a=100$ and $10 \times 10 \times 10$ for $l / a=10$.

Table 3. Dimensionless displacements for a slender simply supported beam under a surface bending load via FEM 1D and FEM PGD 1D solutions, 121 nodes and $N_{c}=6$.

\begin{tabular}{|c|c|c|c|c|c|c|c|c|c|}
\hline & \multicolumn{3}{|c|}{$10^{-2} \times \bar{u}_{x}$} & \multicolumn{3}{|c|}{$10^{-3} \times \bar{u}_{y}$} & \multicolumn{3}{|c|}{$-10 \times \bar{u}_{z}$} \\
\hline & $B 2$ & $B 3$ & $B 4$ & $B 2$ & B3 & $B 4$ & $B 2$ & B3 & $B 4$ \\
\hline \multicolumn{10}{|c|}{ FEM 1D } \\
\hline$N=2-6$ & 1.000 & 1.000 & 1.000 & 6.250 & 6.251 & 6.251 & 4.950 & 4.951 & 4.951 \\
\hline \multicolumn{10}{|c|}{ FEM PGD 1D } \\
\hline$N=6$ & 1.000 & 1.000 & 1.000 & 6.250 & 6.251 & 6.251 & 4.950 & 4.951 & 4.950 \\
\hline$N=4,5$ & 1.000 & 1.000 & 1.000 & 6.250 & 6.252 & 6.251 & 4.950 & 4.956 & 4.950 \\
\hline$N=3$ & 1.000 & 1.000 & 1.000 & 6.250 & 6.251 & 6.251 & 4.950 & 4.951 & 4.951 \\
\hline$N=2$ & 1.000 & 1.000 & 1.000 & 6.250 & 6.251 & 6.251 & 4.950 & 4.951 & 4.950 \\
\hline
\end{tabular}

Table 4. Dimensionless displacements for a short simply supported beam under a surface bending load via FEM 1D and FEM PGD 1D solutions, 121 nodes and $N_{c}=7$.

\begin{tabular}{|c|c|c|c|c|c|c|c|c|c|}
\hline & \multicolumn{3}{|c|}{$10^{-1} \times \bar{u}_{x}$} & \multicolumn{3}{|c|}{$10^{-1} \times \bar{u}_{y}$} & \multicolumn{3}{|c|}{$-10 \times \bar{u}_{z}$} \\
\hline & $B 2$ & $B 3$ & $B 4$ & $B 2$ & B3 & $B 4$ & $B 2$ & $B 3$ & $B 4$ \\
\hline \multicolumn{10}{|c|}{ FEM 1D } \\
\hline$N=6$ & 1.002 & 1.002 & 1.002 & 6.361 & 6.362 & 6.362 & 5.017 & 5.018 & 5.018 \\
\hline$N=5$ & 1.002 & 1.002 & 1.002 & 6.361 & 6.362 & 6.362 & 5.017 & 5.017 & 5.017 \\
\hline$N=4$ & 1.001 & 1.001 & 1.001 & 6.361 & 6.362 & 6.362 & 5.015 & 5.016 & 5.016 \\
\hline$N=3$ & 1.001 & 1.001 & 1.001 & 6.361 & 6.362 & 6.362 & 5.014 & 5.014 & 5.014 \\
\hline$N=2$ & 0.999 & 0.999 & 0.999 & 6.339 & 6.340 & 6.340 & 5.002 & 5.002 & 5.002 \\
\hline \multicolumn{10}{|c|}{ FEM PGD 1D } \\
\hline$N=6$ & 1.002 & 1.002 & 1.002 & 6.361 & 6.362 & 6.362 & 5.017 & 5.017 & 5.017 \\
\hline$N=5$ & 1.002 & 1.002 & 1.002 & 6.361 & 6.362 & 6.362 & 5.016 & 5.017 & 5.016 \\
\hline$N=4$ & 1.001 & 1.001 & 1.001 & 6.361 & 6.362 & 6.362 & 5.015 & 5.015 & 5.015 \\
\hline$N=3$ & 1.001 & 1.001 & 1.001 & 6.361 & 6.362 & 6.362 & 5.014 & 5.015 & 5.015 \\
\hline$N=2$ & 0.999 & 0.999 & 0.999 & 6.339 & 6.340 & 6.340 & 5.002 & 5.002 & 5.002 \\
\hline
\end{tabular}


of the results can be clearly identified. For the presented cases, the displacements computed via the different solution techniques are coincident.

The stresses presented in Tables $5-7$ are put into the following dimensionless form:

$$
\bar{\sigma}_{i j}=\frac{\sigma_{i j}}{p_{y y}}
$$

where $\bar{\sigma}_{x x}$ is computed at $(l / 2,-a / 2,+b / 2), \bar{\sigma}_{y y}$ at $(l / 2,0,+b / 2)$ and $\bar{\sigma}_{x y}$ at $(0,0,+b / 2)$. Accurate results can be obtained by the proposed models as shown

Table 5. Dimensionless stresses for slender and short simply supported beams under a surface bending load via three-dimensional finite element and Navier-type solutions.

\begin{tabular}{|c|c|c|c|c|c|}
\hline & \multicolumn{2}{|c|}{$l / a=100$} & \multicolumn{3}{|c|}{$l / a=10$} \\
\hline & $-10^{-3} \times \bar{\sigma}_{x x}$ & $10^{-1} \times \bar{\sigma}_{x y}$ & $-10^{-1} \times \bar{\sigma}_{x x}$ & $10 \times \bar{\sigma}_{y y}$ & $\bar{\sigma}_{x y}$ \\
\hline FEM 3D-R ${ }^{a}$ & 7.500 & 8.662 & 7.511 & 5.000 & 8.502 \\
\hline FEM 3D-C ${ }^{b}$ & 7.501 & 8.707 & 7.559 & 4.997 & 8.653 \\
\hline$N=9$ & 7.500 & 8.647 & 7.510 & 5.000 & 8.480 \\
\hline$N=8$ & 7.500 & 8.650 & 7.510 & 5.000 & 8.490 \\
\hline$N=7$ & 7.500 & 8.650 & 7.510 & 5.000 & 8.496 \\
\hline$N=6$ & 7.500 & 8.728 & 7.510 & 5.000 & 8.567 \\
\hline$N=5$ & 7.500 & 8.728 & 7.511 & 5.000 & 8.566 \\
\hline$N=4$ & 7.500 & 8.513 & 7.508 & 5.000 & 8.372 \\
\hline$N=3$ & 7.500 & 8.513 & 7.518 & 5.000 & 8.333 \\
\hline$N=2$ & 7.500 & 5.914 & 7.500 & 5.000 & 5.751 \\
\hline
\end{tabular}

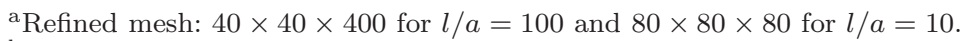

${ }^{\mathrm{b}}$ Coarse mesh: $10 \times 10 \times 100$ for $l / a=100$ and $10 \times 10 \times 10$ for $l / a=10$.

Table 6. Dimensionless stresses for a slender simply supported beam under a surface bending load via FEM 1D and FEM PGD 1D solutions, 121 nodes and $N_{c}=6$.

\begin{tabular}{|c|c|c|c|c|c|c|}
\hline & \multicolumn{3}{|c|}{$-10^{-3} \times \bar{\sigma}_{x x}$} & \multicolumn{3}{|c|}{$10^{-1} \times \bar{\sigma}_{x y}$} \\
\hline & $B 2$ & $B 3$ & $B 4$ & $B 2$ & $B 3$ & $B 4$ \\
\hline \multicolumn{7}{|c|}{ FEM 1D } \\
\hline$N=9$ & 7.499 & 7.501 & 7.500 & 4.727 & 13.78 & 8.719 \\
\hline$N=8$ & 7.499 & 7.501 & 7.500 & 4.731 & 13.79 & 8.723 \\
\hline$N=7$ & 7.499 & 7.501 & 7.500 & 4.731 & 13.79 & 8.723 \\
\hline$N=6$ & 7.499 & 7.501 & 7.500 & 4.808 & 13.87 & 8.800 \\
\hline$N=5$ & 7.499 & 7.501 & 7.500 & 4.808 & 13.87 & 8.800 \\
\hline$N=4$ & 7.499 & 7.501 & 7.500 & 4.595 & 13.66 & 8.586 \\
\hline$N=3$ & 7.499 & 7.502 & 7.500 & 4.594 & 13.66 & 8.586 \\
\hline$N=2$ & 7.498 & 7.501 & 7.500 & 2.000 & 11.06 & 5.985 \\
\hline \multicolumn{7}{|c|}{ FEM PGD 1D } \\
\hline$N=9$ & 7.499 & 7.502 & 7.500 & 4.714 & 13.79 & 8.698 \\
\hline$N=8$ & 7.499 & 7.502 & 7.500 & 4.718 & 13.79 & 8.701 \\
\hline$N=7$ & 7.499 & 7.502 & 7.500 & 4.718 & 13.79 & 8.701 \\
\hline$N=6$ & 7.499 & 7.502 & 7.500 & 4.793 & 13.86 & 8.777 \\
\hline$N=5$ & 7.498 & 7.508 & 7.500 & 4.793 & 13.84 & 8.777 \\
\hline$N=4$ & 7.498 & 7.508 & 7.500 & 4.581 & 13.63 & 8.565 \\
\hline$N=3$ & 7.499 & 7.502 & 7.500 & 4.585 & 13.66 & 8.570 \\
\hline$N=2$ & 7.498 & 7.501 & 7.500 & 2.000 & 11.05 & 5.984 \\
\hline
\end{tabular}


Table 7. Dimensionless stresses for a deep simply supported beam under a surface bending load via FEM 1D and FEM PGD 1D solutions, 121 nodes and $N_{c}=9$.

\begin{tabular}{|c|c|c|c|c|c|c|c|c|c|}
\hline & \multicolumn{3}{|c|}{$-10^{-1} \times \bar{\sigma}_{x x}$} & \multicolumn{3}{|c|}{$10 \times \bar{\sigma}_{y y}$} & \multicolumn{3}{|c|}{$\bar{\sigma}_{x y}$} \\
\hline & $B 2$ & $B 3$ & $B 4$ & $B 2$ & $B 3$ & $B 4$ & $B 2$ & $B 3$ & $B 4$ \\
\hline \multicolumn{10}{|c|}{ FEM 1D } \\
\hline$N=9$ & 7.508 & 7.511 & 7.510 & 5.000 & 5.000 & 5.000 & 8.437 & 8.546 & 8.483 \\
\hline$N=8$ & 7.508 & 7.511 & 7.510 & 5.000 & 5.000 & 5.000 & 8.447 & 8.556 & 8.493 \\
\hline$N=7$ & 7.508 & 7.511 & 7.510 & 5.000 & 5.000 & 5.000 & 8.449 & 8.560 & 8.500 \\
\hline$N=6$ & 7.509 & 7.512 & 7.510 & 5.000 & 5.000 & 5.000 & 8.521 & 8.632 & 8.572 \\
\hline$N=5$ & 7.510 & 7.513 & 7.511 & 5.000 & 5.000 & 5.000 & 8.513 & 8.622 & 8.561 \\
\hline$N=4$ & 7.507 & 7.510 & 7.508 & 5.000 & 5.000 & 5.000 & 8.320 & 8.427 & 8.367 \\
\hline$N=3$ & 7.517 & 7.520 & 7.518 & 5.000 & 5.000 & 5.000 & 8.308 & 8.408 & 8.336 \\
\hline$N=2$ & 7.498 & 7.502 & 7.500 & 5.000 & 5.000 & 5.000 & 5.726 & 5.824 & 5.754 \\
\hline \multicolumn{10}{|c|}{ FEM PGD 1D } \\
\hline$N=9$ & 7.509 & 7.512 & 7.511 & 5.003 & 5.002 & 5.007 & 8.437 & 8.547 & 8.488 \\
\hline$N=8$ & 7.509 & 7.512 & 7.510 & 5.011 & 5.007 & 5.010 & 8.449 & 8.558 & 8.496 \\
\hline$N=7$ & 7.509 & 7.512 & 7.510 & 5.019 & 5.008 & 5.008 & 8.449 & 8.556 & 8.495 \\
\hline$N=6$ & 7.509 & 7.513 & 7.511 & 5.006 & 5.010 & 5.006 & 8.524 & 8.634 & 8.574 \\
\hline$N=5$ & 7.510 & 7.514 & 7.512 & 5.010 & 5.009 & 5.009 & 8.515 & 8.625 & 8.564 \\
\hline$N=4$ & 7.507 & 7.510 & 7.508 & 5.016 & 5.017 & 5.016 & 8.320 & 8.432 & 8.371 \\
\hline$N=3$ & 7.517 & 7.520 & 7.518 & 5.000 & 4.997 & 4.997 & 8.310 & 8.414 & 8.344 \\
\hline$N=2$ & 7.499 & 7.502 & 7.500 & 5.001 & 5.001 & 5.002 & 5.727 & 5.824 & 5.754 \\
\hline
\end{tabular}

by Table 5. FEM PGD results are in good agreement with the classical finite element ones. The finite approximation of the shear stress component $\sigma_{x y}$ via linear and quadratic elements for $l / a=100$ is not accurate for the considered number of nodes. Accuracy can be improved by either increasing the number of nodes or by using a stress recovery technique such as the integration of the indefinite equilibrium equations [Tornabene et al., 2012].

Figure 8 presents the though-the-thickness variation of the normal stress components $\bar{\sigma}_{y y}$ and $\bar{\sigma}_{z z}$ for a short beam. Results are computed at mid span for $z / b=0.5$

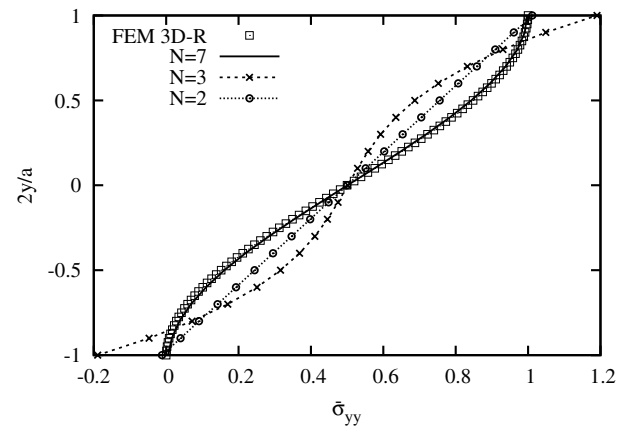

(a)

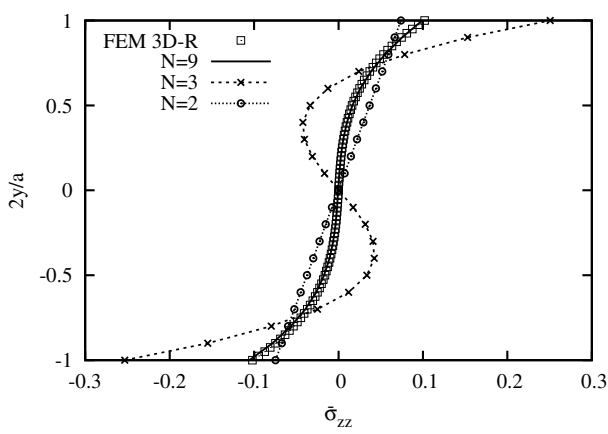

(b)

Fig. 8. Normal stresses variation over the cross-section: (a) $\bar{\sigma}_{y y}(l / 2, y, b / 2)$ and (b) $\bar{\sigma}_{z z}(l / 2, y, 0)$. Simply supported beam, $l / a=10$, cubic elements and $N_{c}=7$. 


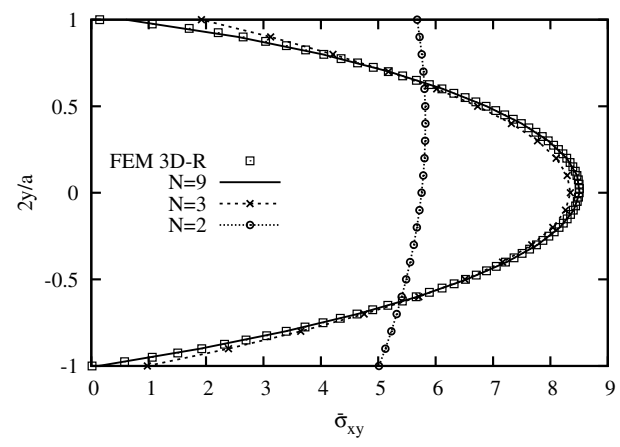

(a)

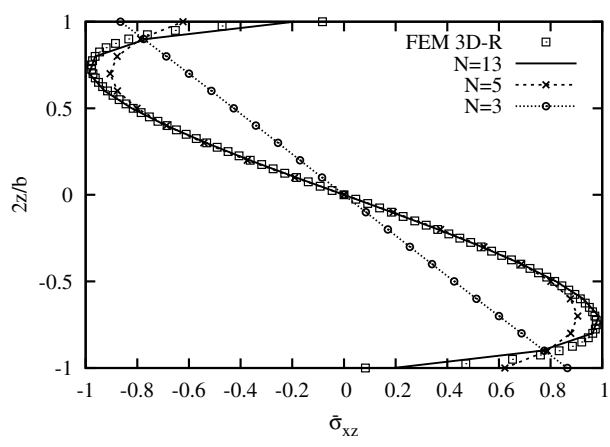

(b)

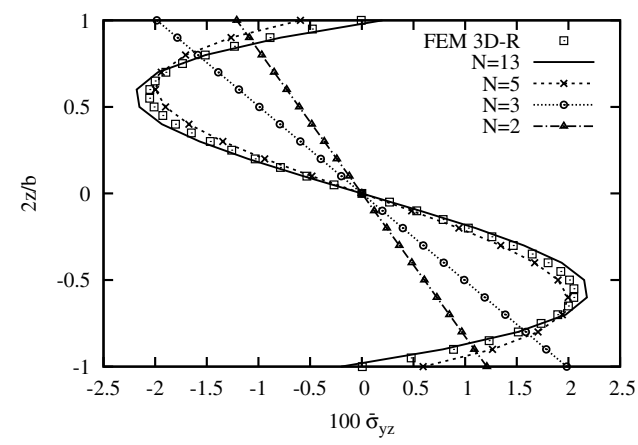

(c)

Fig. 9. Shear stresses variation over the cross-section: (a) $\bar{\sigma}_{x y}(0, y,+b / 2)$, (b) $\bar{\sigma}_{x z}(0, a / 2, z)$ and (c) $\bar{\sigma}_{y z}(l / 2,0, z)$. Simply supported beam, $l / a=10$, cubic elements and $N_{c}=7$.

and zero, respectively. Stress component $\bar{\sigma}_{x x}$ is not reported for the sake of brevity. It is linear and a second-order model is equal to the three-dimensional finite element solution. Shear stress components $\bar{\sigma}_{x y}, \bar{\sigma}_{x z}$ and $\bar{\sigma}_{y z}$ are plotted in Fig. 9. An accurate three-dimensional stress field can be obtained by the proposed models within a PGD FEM framework as long as an appropriate cross-section expansion order is considered.

As far as the computational costs are concerned, the dof of a three-dimensional finite element solution using serendipity Lagrangian elements are:

$$
\operatorname{dof}_{\mathrm{FEM} \mathrm{3D}}=3\left[N_{e x}\left(4 N_{e y}^{2}+6 N_{e y}+2\right)+3 N_{e y}^{2}+4 N_{e y}+1\right],
$$

where $N_{e x}$ and $N_{e y}$ are the number of elements along $x$ and $y$ and it is assumed that the number of elements along $z$ are equal to $N_{e y}$. The dof of the Naviertype solution represent the number of unknowns per cross-section and they are $\frac{3}{2}(N+1)(N+2)$. The number of dofs of a classical FEM 1D based upon the proposed beam models is $\frac{1}{3} N_{\mathcal{D}_{\Omega}} \cdot N_{\mathcal{D}_{x}}$ where $N_{\mathcal{D}_{\Omega}}$ and $N_{\mathcal{D}_{x}}$ have been defined in Eqs. (51) and (55), respectively. In the case of a FEM PGD 1D solution, the number of dofs 
Table 8. Computational costs of the considered solutions for a short simply supported beam.

\begin{tabular}{lr}
\hline & \multicolumn{1}{c}{ dofs } \\
\hline FEM 3D-R $(80 \times 80 \times 80)$ & $6^{\prime} 318^{\prime} 243$ \\
FEM 3D-C $(10 \times 10 \times 10)$ & $14^{\prime} 883$ \\
Navier solution $N=9$ & 165 \\
Navier solution $N=2$ & 18 \\
FEM 1D $N=9,121$ nodes & $19^{\prime} 965$ \\
FEM 1D $N=2,121$ nodes & $2^{\prime} 178$ \\
FEM PGD 1D $N=9,121$ nodes & 528 \\
FEM PGD 1D $N=2,121$ nodes & 381 \\
\hline
\end{tabular}

are $N_{\mathcal{D}_{\Omega}}+N_{\mathcal{D}_{x}}$. Table 8 presents the computational cost of the solutions used in this paper showing that this method is very effective in reducing the dimension of the global problem. As far as the computational time is concerned, the two solutions are comparable for the considered number couples (of the order of few seconds). A FEM PGD solution can require more time than a classical FEM on as soon as $N_{c}$ increases. Time can be reduced significantly reduced by exploiting in the code implementation the matrices sparsity and parallelization for all the vector-matrix multiplications.

Tables 9 and 10 present the case of clamped-clamped short beams. Displacements $\bar{u}_{x}, \bar{u}_{y}$ and $\bar{u}_{z}$ are evaluated at $(l / 4,-a / 2,+b / 2),(l / 2,+a / 2,0)$ and $(l / 2,-a / 2,+b / 2)$, respectively. Stresses $\bar{\sigma}_{x x}, \bar{\sigma}_{y y}$ and $\bar{\sigma}_{x y}$ are calculated at $(l / 2,+a / 2,+b / 2),(l / 2,+a / 2,0)$ and $(l / 4,0,0)$. The FEM $1 D$ s have been obtained by 181 nodes. For the PGD results, 13 couples are built. The results are all in good

Table 9. Dimensionless displacements for a short clamped-clamped beam under a surface bending load via FEM 3D, FEM 1D and FEM PGD 1D solutions, 181 nodes and $N_{c}=13$.

\begin{tabular}{|c|c|c|c|c|c|c|c|c|c|}
\hline & \multicolumn{3}{|c|}{$\bar{u}_{x}$} & \multicolumn{3}{|c|}{$10^{-1} \times \bar{u}_{y}$} & \multicolumn{3}{|c|}{$10 \times \bar{u}_{z}$} \\
\hline FEM 3D-R ${ }^{\mathrm{a}}$ & \multicolumn{3}{|c|}{1.861} & \multicolumn{3}{|c|}{1.372} & \multicolumn{3}{|c|}{1.636} \\
\hline FEM $3 \mathrm{D}-\mathrm{C}^{\mathrm{b}}$ & \multicolumn{3}{|c|}{1.852} & \multicolumn{3}{|c|}{1.369} & \multicolumn{3}{|c|}{1.814} \\
\hline FEM 1D & $B 2$ & $B 3$ & $B 4$ & $B 2$ & B3 & $B 4$ & $B 2$ & $B 3$ & $B 4$ \\
\hline$N=9$ & 1.859 & 1.873 & 1.860 & 1.370 & 1.371 & 1.371 & 1.635 & 1.636 & 1.636 \\
\hline$N=7$ & 1.859 & 1.872 & 1.860 & 1.370 & 1.371 & 1.371 & 1.635 & 1.635 & 1.636 \\
\hline$N=5$ & 1.858 & 1.872 & 1.859 & 1.369 & 1.370 & 1.370 & 1.633 & 1.634 & 1.634 \\
\hline$N=4$ & 1.855 & 1.868 & 1.856 & 1.368 & 1.369 & 1.369 & 1.631 & 1.632 & 1.632 \\
\hline$N=3$ & 1.852 & 1.865 & 1.852 & 1.364 & 1.365 & 1.365 & 1.628 & 1.628 & 1.628 \\
\hline$N=2$ & 1.840 & 1.852 & 1.840 & 1.342 & 1.343 & 1.343 & 1.614 & 1.615 & 1.615 \\
\hline FEM PGD 1D & $B 2$ & $B 3$ & $B 4$ & $B 2$ & $B 3$ & $B 4$ & $B 2$ & $B 3$ & $B 4$ \\
\hline$N=9$ & 1.856 & 1.870 & 1.857 & 1.368 & 1.369 & 1.369 & 1.636 & 1.637 & 1.637 \\
\hline$N=7$ & 1.856 & 1.869 & 1.857 & 1.368 & 1.368 & 1.369 & 1.630 & 1.631 & 1.631 \\
\hline$N=5$ & 1.858 & 1.871 & 1.859 & 1.368 & 1.369 & 1.369 & 1.630 & 1.631 & 1.631 \\
\hline$N=4$ & 1.854 & 1.867 & 1.849 & 1.367 & 1.368 & 1.364 & 1.628 & 1.628 & 1.621 \\
\hline$N=3$ & 1.851 & 1.864 & 1.852 & 1.364 & 1.364 & 1.364 & 1.627 & 1.628 & 1.628 \\
\hline$N=2$ & 1.839 & 1.852 & 1.840 & 1.342 & 1.343 & 1.343 & 1.614 & 1.615 & 1.615 \\
\hline
\end{tabular}

a Mesh: $80 \times 80 \times 80 .{ }^{\mathrm{b}}$ Mesh: $12 \times 12 \times 12$. 
Table 10. Dimensionless stresses for a short clamped-clamped beam under a surface bending load via FEM 3D, FEM 1D and FEM PGD 1D solutions, 181 nodes and $N_{c}=13$.

\begin{tabular}{lccccccccc}
\hline & \multicolumn{3}{c}{$10^{-1} \times \bar{\sigma}_{x x}$} & \multicolumn{3}{c}{$\bar{\sigma}_{y y}$} & \multicolumn{3}{c}{$\bar{\sigma}_{x y}$} \\
\hline FEM 3D-R $^{\mathrm{a}}$ & \multicolumn{3}{c}{2.519} & & & 1.000 & & & 3.472 \\
FEM 3D-C & & 2.569 & & & 1.136 & & & 3.472 & \\
FEM 1D & $B 2$ & $B 3$ & $B 4$ & $B 2$ & $B 3$ & $B 4$ & $B 2$ & $B 3$ & $B 4$ \\
$N=9$ & 2.516 & 2.518 & 2.518 & 0.998 & 1.001 & 1.000 & 3.306 & 3.560 & 3.472 \\
$N=7$ & 2.516 & 2.518 & 2.517 & 0.995 & 0.999 & 0.997 & 3.308 & 3.562 & 3.474 \\
$N=5$ & 2.517 & 2.519 & 2.518 & 0.980 & 0.985 & 0.982 & 3.304 & 3.558 & 3.469 \\
$N=4$ & 2.512 & 2.514 & 2.514 & 1.002 & 1.007 & 1.004 & 3.327 & 3.581 & 3.492 \\
$N=3$ & 2.519 & 2.521 & 2.520 & 1.308 & 1.314 & 1.310 & 3.327 & 3.580 & 3.492 \\
$N=2$ & 2.499 & 2.501 & 2.500 & 1.010 & 1.017 & 1.012 & 2.341 & 2.567 & 2.500 \\
FEM PGD 1D & $B 2$ & $B 3$ & $B 4$ & $B 2$ & $B 3$ & $B 4$ & $B 2$ & $B 3$ & $B 4$ \\
$N=9$ & 2.511 & 2.512 & 2.511 & 0.969 & 0.974 & 0.978 & 3.316 & 3.569 & 3.481 \\
$N=7$ & 2.513 & 2.515 & 2.514 & 0.959 & 0.960 & 0.959 & 3.315 & 3.570 & 3.481 \\
$N=5$ & 2.515 & 2.517 & 2.516 & 0.980 & 0.982 & 0.975 & 3.303 & 3.557 & 3.467 \\
$N=4$ & 2.511 & 2.513 & 2.504 & 1.023 & 1.016 & 1.023 & 3.330 & 3.584 & 3.484 \\
$N=3$ & 2.518 & 2.520 & 2.519 & 1.302 & 1.309 & 1.304 & 3.328 & 3.582 & 3.493 \\
$N=2$ & 2.499 & 2.501 & 2.500 & 1.010 & 1.017 & 1.012 & 2.341 & 2.567 & 2.500 \\
\hline
\end{tabular}

${ }^{\mathrm{a}}$ Mesh: $80 \times 80 \times 80 .{ }^{\mathrm{b}} \mathrm{Mesh}: 12 \times 12 \times 12$.

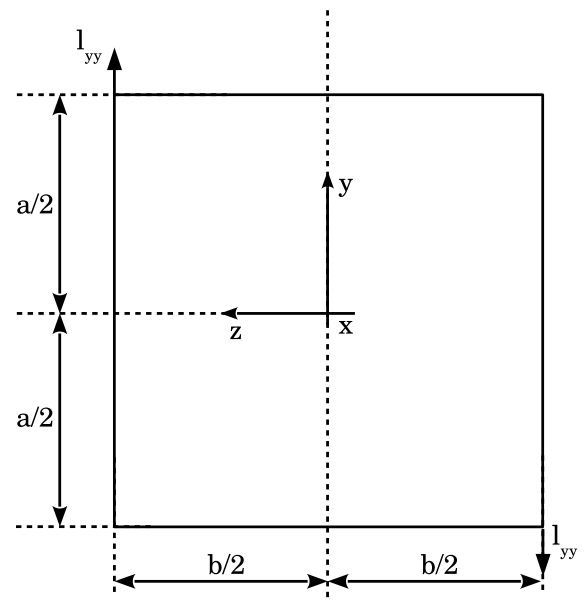

Fig. 10. Square cross-section geometry and line torsional loads.

agreement showing the effectiveness of the one-dimensional modeling within a PGD framework.

\subsection{Square cross-section beams under torsion}

Two line loads $l_{y y}$ equal to $1 \mathrm{~N} / \mathrm{m}$ are applied to the beams as presented in Fig. 10. For the sake of brevity, only short beams are considered. The considered 
displacements and stresses are put into the following dimensionless form:

$$
\begin{aligned}
\bar{u}_{y} & =4 \frac{a b}{l^{2}} \frac{E}{l_{y y}} u_{y}\left(\frac{l}{2},+\frac{a}{2},+\frac{b}{2}\right), \\
\bar{u}_{z} & =4 \frac{a b}{l^{2}} \frac{E}{l_{y y}} u_{z}\left(\frac{l}{2},-\frac{a}{2},-\frac{b}{2}\right), \\
\bar{\sigma}_{x y} & =\frac{b}{l_{y y}} \sigma_{x y}\left(0,0,+\frac{b}{2}\right), \\
\bar{\sigma}_{x z} & =\frac{b}{l_{y y}} \sigma_{x z}\left(0,-\frac{a}{2}, 0\right) .
\end{aligned}
$$

Tables 11-13 present the case of a simply supported beam. As far as the proposed models are concerned, Table 11 shows that low-order theories are inadequate since

Table 11. Dimensionless displacements and stresses for a short simply supported beam under torsional line loads via three-dimensional finite element and Navier-type solutions.

\begin{tabular}{lcccc}
\hline & $\bar{u}_{y}$ & $\bar{u}_{z}$ & $10^{-1} \times \bar{\sigma}_{x y}$ & $10^{-1} \times \bar{\sigma}_{x z}$ \\
\hline FEM 3D-R $^{\mathrm{a}}$ & 5.166 & 4.987 & 2.373 & 2.341 \\
FEM 3D-C $^{\mathrm{b}}$ & 5.004 & 4.899 & 2.400 & 2.372 \\
$N=11$ & 5.002 & 4.875 & 2.385 & 2.340 \\
$N=9$ & 4.977 & 4.859 & 2.344 & 2.291 \\
$N=7$ & 4.941 & 4.835 & 2.373 & 2.379 \\
$N=5$ & 4.899 & 4.808 & 2.431 & 2.431 \\
$N=4$ & 4.846 & 4.776 & 2.508 & 2.408 \\
$N=3$ & 4.107 & 4.038 & 1.538 & 1.456 \\
$N=2$ & 4.023 & 3.983 & 1.534 & 1.457 \\
\hline
\end{tabular}

${ }^{\mathrm{a}}$ Refined mesh: $60 \times 60 \times 60$. ${ }^{\mathrm{b}}$ Coarse mesh: $10 \times 10 \times 10$.

\begin{tabular}{|c|c|c|c|c|c|c|}
\hline & & $\bar{u}_{y}$ & & & $\bar{u}_{z}$ & \\
\hline FEM 1D & $B 2$ & $B 3$ & $B 4$ & $B 2$ & $B 3$ & $B 4$ \\
\hline$N=11$ & 5.003 & 5.003 & 5.003 & 4.876 & 4.876 & 4.876 \\
\hline$N=9$ & 4.976 & 4.976 & 4.976 & 4.859 & 4.859 & 4.859 \\
\hline$N=7$ & 4.941 & 4.941 & 4.941 & 4.835 & 4.835 & 4.835 \\
\hline$N=5$ & 4.899 & 4.899 & 4.899 & 4.808 & 4.808 & 4.808 \\
\hline$N=4$ & 4.846 & 4.846 & 4.846 & 4.776 & 4.776 & 4.776 \\
\hline$N=3$ & 4.107 & 4.107 & 4.107 & 4.038 & 4.038 & 4.038 \\
\hline$N=2$ & 4.023 & 4.023 & 4.023 & 3.983 & 3.983 & 3.983 \\
\hline FEM PGD 1D & $B 2$ & $B 3$ & $B 4$ & $B 2$ & $B 3$ & $B 4$ \\
\hline$N=11$ & 5.003 & 5.003 & 5.003 & 4.876 & 4.876 & 4.876 \\
\hline$N=9$ & 4.977 & 4.977 & 4.976 & 4.859 & 4.859 & 4.859 \\
\hline$N=7$ & 4.941 & 4.941 & 4.941 & 4.836 & 4.836 & 4.836 \\
\hline$N=5$ & 4.895 & 4.895 & 4.895 & 4.804 & 4.804 & 4.804 \\
\hline$N=4$ & 4.846 & 4.846 & 4.846 & 4.776 & 4.776 & 4.777 \\
\hline$N=3$ & 4.106 & 4.106 & 4.106 & 4.036 & 4.036 & 4.036 \\
\hline$N=2$ & 4.023 & 4.023 & 4.023 & 3.983 & 3.983 & 3.983 \\
\hline
\end{tabular}

Table 12. Dimensionless displacements for a short simply supported beam under torsional line loads via FEM 3D, FEM 1D and FEM PGD 1D solutions, 181 nodes and $N_{c}=9$. 
Table 13. Dimensionless shear stresses for a short simply supported beam under torsional line loads via FEM 3D, FEM 1D and FEM PGD 1D solutions, 181 nodes and $N_{c}=9$.

\begin{tabular}{lcccccc}
\hline & \multicolumn{3}{c}{$10^{-1} \times \bar{\sigma}_{x y}$} & \multicolumn{3}{c}{$10^{-1} \times \bar{\sigma}_{x z}$} \\
\hline FEM 1D & $B 2$ & $B 3$ & $B 4$ & $B 2$ & $B 3$ & $B 4$ \\
$N=11$ & 2.384 & 2.390 & 2.389 & 2.339 & 2.343 & 2.341 \\
$N=9$ & 2.340 & 2.347 & 2.347 & 2.289 & 2.290 & 2.290 \\
$N=7$ & 2.374 & 2.372 & 2.370 & 2.379 & 2.380 & 2.379 \\
$N=5$ & 2.434 & 2.431 & 2.429 & 2.431 & 2.433 & 2.432 \\
$N=4$ & 2.503 & 2.516 & 2.516 & 2.406 & 2.409 & 2.408 \\
$N=3$ & 1.532 & 1.545 & 1.546 & 1.454 & 1.457 & 1.456 \\
$N=2$ & 1.527 & 1.542 & 1.543 & 1.456 & 1.458 & 1.457 \\
FEM PGD 1D & $B 2$ & $B 3$ & $B 4$ & $B 2$ & $B 3$ & $B 4$ \\
$N=11$ & 2.384 & 2.390 & 2.387 & 2.340 & 2.343 & 2.341 \\
$N=9$ & 2.340 & 2.346 & 2.345 & 2.289 & 2.289 & 2.288 \\
$N=7$ & 2.369 & 2.363 & 2.357 & 2.381 & 2.383 & 2.382 \\
$N=5$ & 2.427 & 2.420 & 2.416 & 2.429 & 2.431 & 2.430 \\
$N=4$ & 2.503 & 2.516 & 2.517 & 2.406 & 2.409 & 2.408 \\
$N=3$ & 1.532 & 1.545 & 1.545 & 1.453 & 1.456 & 1.455 \\
$N=2$ & 1.527 & 1.542 & 1.543 & 1.456 & 1.458 & 1.457 \\
\hline
\end{tabular}

the cross-section is too stiff on its plane. Higher-order theories yield fairly good results compared to the FEM 3D-R reference solution. Classical and PGD FEM 1Ds, see Tables 12 and 13, have been computed using 181 nodes along the beam axis and $N_{c}=9$. These solutions match the Navier-type results demonstrating that they can provide accurate results. These methods are also more general since there

Table 14. Dimensionless displacements for a short clamped-clamped beam under torsional line loads via FEM 3D, 1D and PGD 1D solutions, 181 nodes and $N_{c}=9$.

\begin{tabular}{|c|c|c|c|c|c|c|}
\hline & \multicolumn{3}{|c|}{$\bar{u}_{y}$} & \multicolumn{3}{|c|}{$\bar{u}_{z}$} \\
\hline FEM 3D-R ${ }^{a}$ & & 5.131 & & & 4.957 & \\
\hline FEM 3D-C ${ }^{b}$ & & 4.974 & & & 4.874 & \\
\hline FEM 1D & $B 2$ & $B 3$ & $B 4$ & $B 2$ & $B 3$ & $B 4$ \\
\hline$N=11$ & 4.968 & 4.968 & 4.968 & 4.845 & 4.845 & 4.845 \\
\hline$N=9$ & 4.941 & 4.941 & 4.941 & 4.828 & 4.828 & 4.828 \\
\hline$N=7$ & 4.906 & 4.906 & 4.906 & 4.804 & 4.804 & 4.804 \\
\hline$N=5$ & 4.858 & 4.858 & 4.858 & 4.771 & 4.771 & 4.771 \\
\hline$N=4$ & 4.799 & 4.799 & 4.799 & 4.733 & 4.733 & 4.733 \\
\hline$N=3$ & 4.105 & 4.105 & 4.105 & 4.040 & 4.040 & 4.040 \\
\hline$N=2$ & 4.021 & 4.021 & 4.021 & 3.985 & 3.985 & 3.985 \\
\hline FEM PGD 1D & $B 2$ & $B 3$ & $B 4$ & $B 2$ & $B 3$ & $B 4$ \\
\hline$N=11$ & 4.968 & 4.968 & 4.968 & 4.845 & 4.845 & 4.845 \\
\hline$N=9$ & 4.941 & 4.922 & 4.941 & 4.827 & 4.809 & 4.828 \\
\hline$N=7$ & 4.906 & 4.906 & 4.906 & 4.804 & 4.804 & 4.804 \\
\hline$N=5$ & 4.858 & 4.858 & 4.858 & 4.771 & 4.771 & 4.771 \\
\hline$N=4$ & 4.798 & 4.798 & 4.798 & 4.733 & 4.733 & 4.733 \\
\hline$N=3$ & 4.105 & 4.105 & 4.105 & 4.039 & 4.039 & 4.039 \\
\hline$N=2$ & 4.021 & 4.021 & 4.021 & 3.985 & 3.985 & 3.985 \\
\hline
\end{tabular}

a Refined mesh: $60 \times 60 \times 60$. ${ }^{\mathrm{b}}$ Coarse mesh: $10 \times 10 \times 10$. 
Table 15. Dimensionless shear stresses for a short clamped-clamped beam under torsional line loads via FEM 3D, 1D and PGD 1D solutions, 181 nodes and $N_{c}=9$.

\begin{tabular}{lcccccc}
\hline & \multicolumn{3}{c}{$10^{-1} \times \bar{\sigma}_{x y}$} & \multicolumn{3}{c}{$10^{-1} \times \bar{\sigma}_{x z}$} \\
\hline FEM 3D-R ${ }^{\mathrm{a}}$ & \multicolumn{3}{c}{1.928} & & & 1.891 \\
FEM 3D-C & & 1.967 & & & 1.937 & \\
FEM 1D & $B 2$ & $B 3$ & $B 4$ & $B 2$ & $B 3$ & $B 4$ \\
$N=11$ & 1.854 & 1.932 & 1.971 & 1.809 & 1.884 & 1.921 \\
$N=9$ & 1.830 & 1.904 & 1.943 & 1.769 & 1.837 & 1.874 \\
$N=7$ & 1.761 & 1.800 & 1.811 & 1.752 & 1.800 & 1.816 \\
$N=5$ & 1.956 & 2.106 & 2.200 & 1.952 & 2.107 & 2.200 \\
$N=4$ & 1.752 & 1.800 & 1.825 & 1.669 & 1.708 & 1.731 \\
$N=3$ & 1.534 & 1.546 & 1.546 & 1.453 & 1.457 & 1.456 \\
$N=2$ & 1.543 & 1.557 & 1.558 & 1.440 & 1.443 & 1.442 \\
FEM PGD 1D & $B 2$ & $B 3$ & $B 4$ & $B 2$ & $B 3$ & $B 4$ \\
$N=11$ & 1.853 & 1.931 & 1.971 & 1.801 & 1.874 & 1.907 \\
$N=9$ & 1.830 & 1.904 & 1.934 & 1.769 & 1.832 & 1.876 \\
$N=7$ & 1.763 & 1.803 & 1.814 & 1.753 & 1.800 & 1.816 \\
$N=5$ & 1.954 & 2.104 & 2.198 & 1.951 & 2.106 & 2.199 \\
$N=4$ & 1.752 & 1.800 & 1.824 & 1.669 & 1.708 & 1.729 \\
$N=3$ & 1.533 & 1.546 & 1.545 & 1.453 & 1.456 & 1.455 \\
$N=2$ & 1.543 & 1.557 & 1.558 & 1.440 & 1.443 & 1.442 \\
\hline
\end{tabular}

a Refined mesh: $60 \times 60 \times 60 .{ }^{\mathrm{b}}$ Coarse mesh: $10 \times 10 \times 10$.

are no restrictions on the type of displacements' boundary conditions, whereas the Navier-type solution is valid for simply supported beams only.

The dimensionless cross-section displacements and shear stresses for a clampedclamped beam are presented in Tables 14 and 15. FEM three-dimensional, FEM one-dimensional and PGD FEM one-dimensional solutions are compared and the same conclusions drawn for the simply-supported beam are valid in this case.

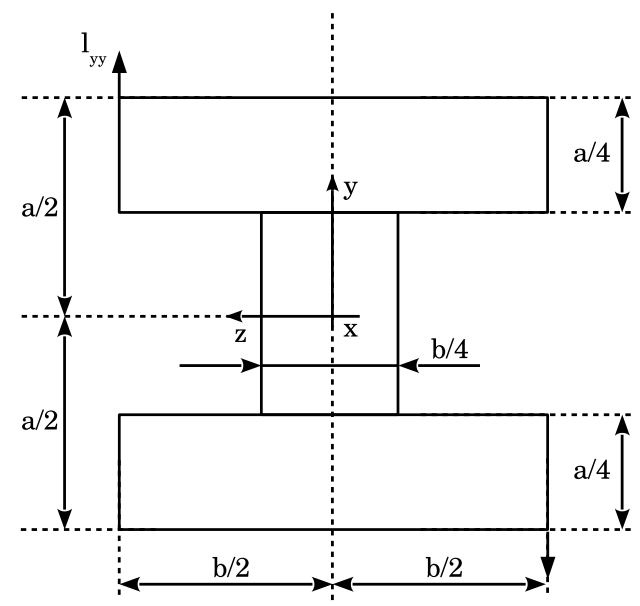

Fig. 11. I-shaped cross-section geometry and line load. 


\subsection{I-shaped cross-section beams under bending and torsion}

An I-shaped beam under a line load is finally investigated. Geometry and load position are shown in Fig. 11. The load is such that it generates bending, torsion and localized effect within the structures. The sharp corners within the cross-section, which in an actual design are avoided, trigger stress concentration. This case is investigated to severely test the accuracy of the proposed one-dimensional approach. The beam is short ( $l / a$ equal to 10$)$ and simply-supported. Three-dimensional finite element solution (mesh: $60 \times 60 \times 60$ ) and the PGD one-dimensional results are qualitatively compared by means of color maps over a cross section $(x / l$ equal to either zero or 0.5 ) where the evaluated quantities assume the maximal value. A solution with $N=17$ and $N_{c}=7$ with cubic elements is considered, 181 nodes along

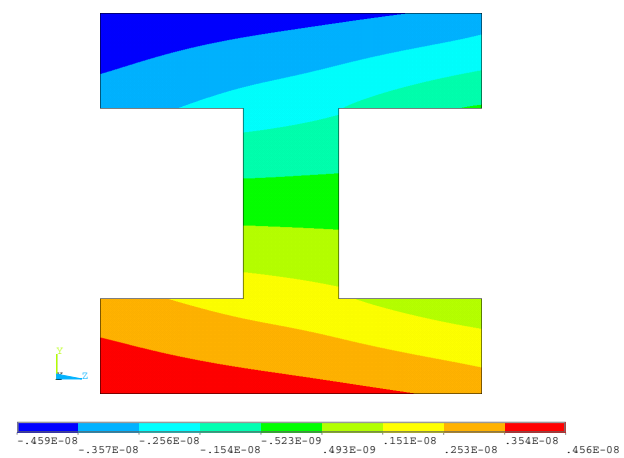

(a)

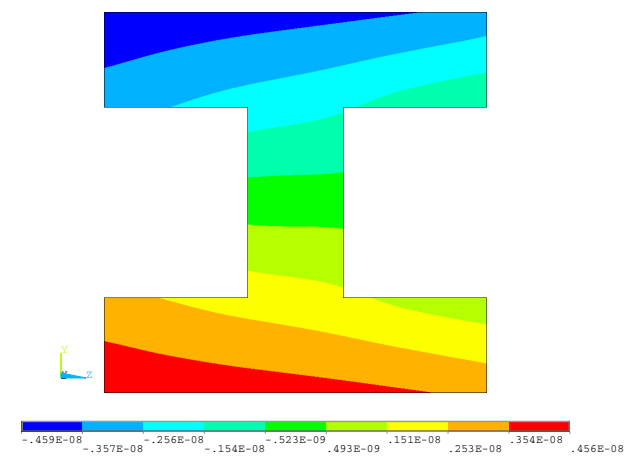

(b)

Fig. 12. Axial displacement $u_{x}[\mathrm{~m}]$ over the cross-section at $x / l=0$ via (a) FEM 3D-R and (b) PGD FEM 1D (cubic elements, $N=17$ and $N_{c}=7$ ) solutions. Simply supported beam with $l / a=10$.

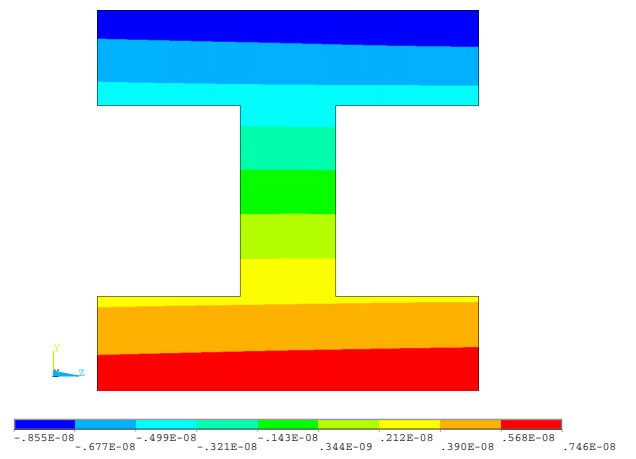

(a)

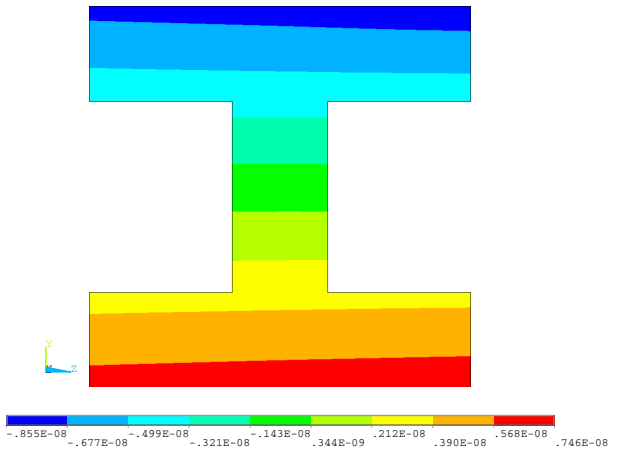

(b)

Fig. 13. Transverse displacement $u_{z}[\mathrm{~m}]$ over the cross-section at $x / l=0.5$ via (a) FEM 3D-R and (b) PGD FEM 1D (cubic elements, $N=17$ and $N_{c}=7$ ) solutions. Simply supported beam with $l / a=10$. 


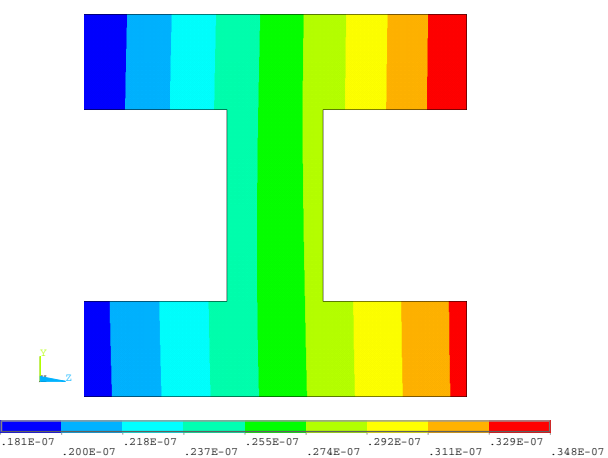

(a)

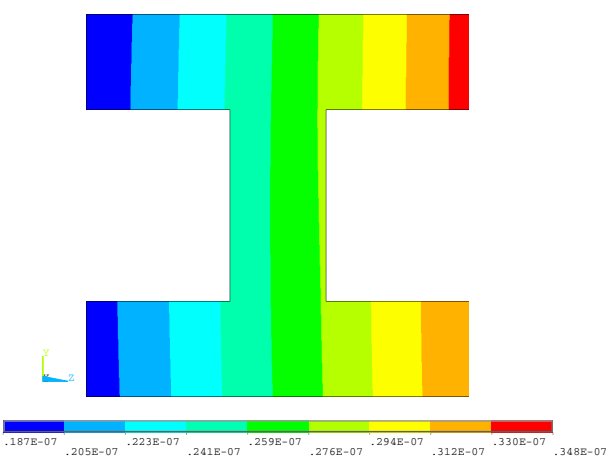

(b)

Fig. 14. Transverse displacement $u_{y}[\mathrm{~m}]$ over the cross-section at $x / l=0.5$ via (a) FEM 3D-R and (b) PGD FEM 1D (cubic elements, $N=17$ and $N_{c}=7$ ) solutions. Simply supported beam with $l / a=10$.

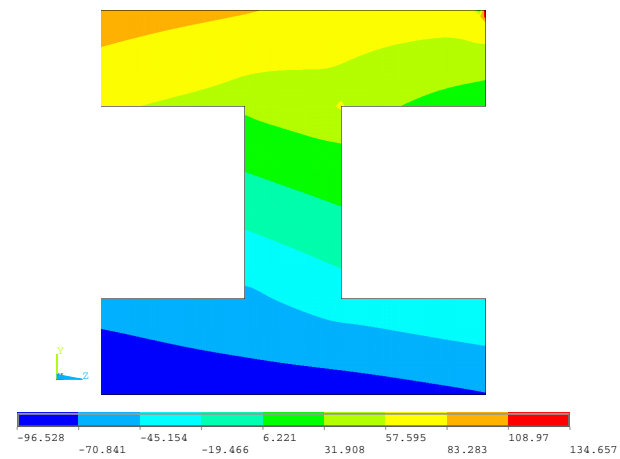

(a)

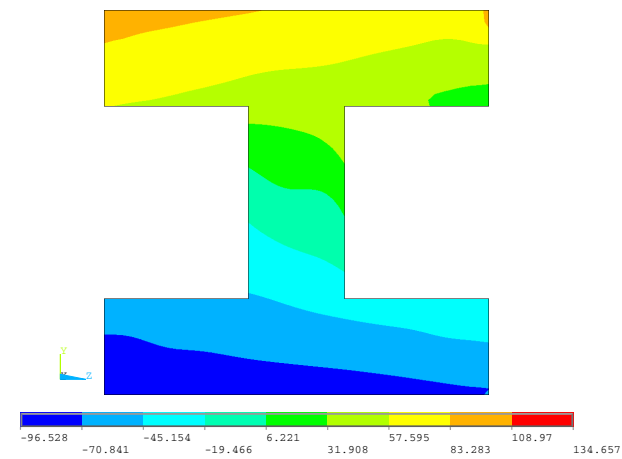

(b)

Fig. 15. Axial stress $\sigma_{x x}[\mathrm{MPa}]$ over the cross-section at $x / l=0.5$ via (a) FEM 3D-R and (b) PGD FEM 1D (cubic elements, $N=17$ and $N_{c}=7$ ) solutions. Simply supported beam with $l / a=10$.

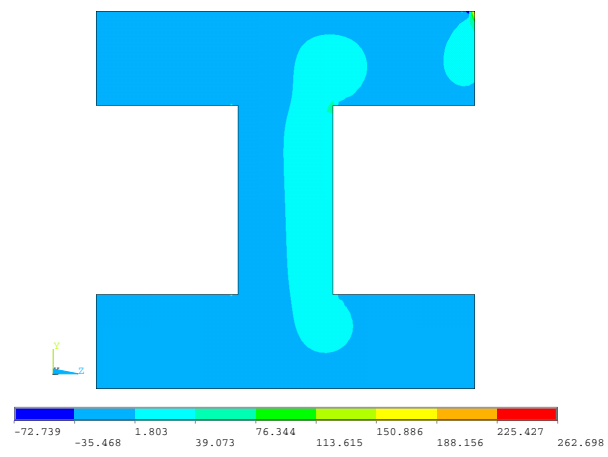

(a)

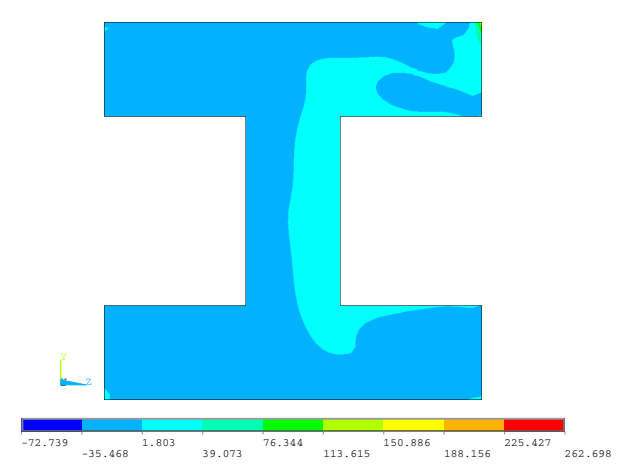

(b)

Fig. 16. Normal stress $\sigma_{y y}[\mathrm{MPa}]$ over the cross-section at $x / l=0.5$ via (a) FEM $3 \mathrm{D}-\mathrm{R}$ and (b) PGD FEM 1D (cubic elements, $N=17$ and $N_{c}=7$ ) solutions. Simply supported beam with $l / a=10$. 


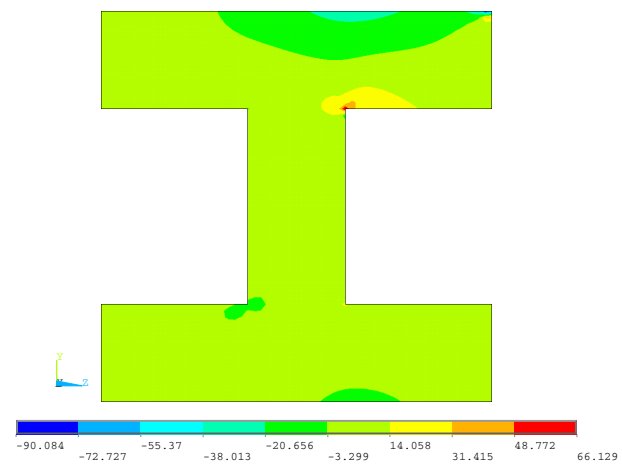

(a)

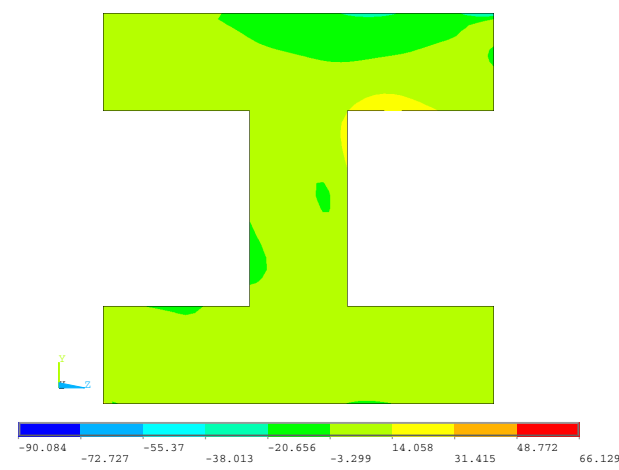

(b)

Fig. 17. Normal stress $\sigma_{z z}[\mathrm{MPa}]$ over the cross-section at $x / l=0.5$ via (a) FEM 3D-R and (b) PGD FEM 1D (cubic elements, $N=17$ and $N_{c}=7$ ) solutions. Simply supported beam with $l / a=10$.

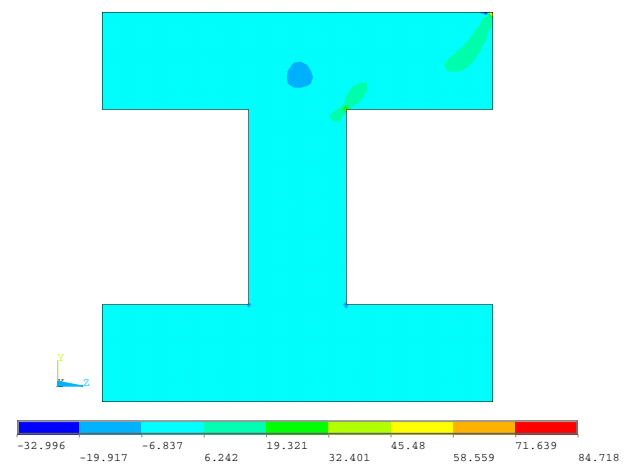

(a)

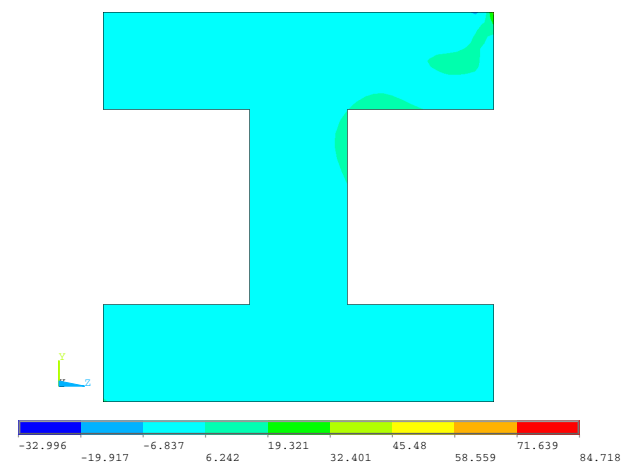

(b)

Fig. 18. Shear stress $\sigma_{y z}[\mathrm{MPa}]$ over the cross-section at $x / l=0.5$ via (a) FEM 3D-R and (b) PGD FEM 1D (cubic elements, $N=17$ and $N_{c}=7$ ) solutions. Simply supported beam with $l / a=10$.

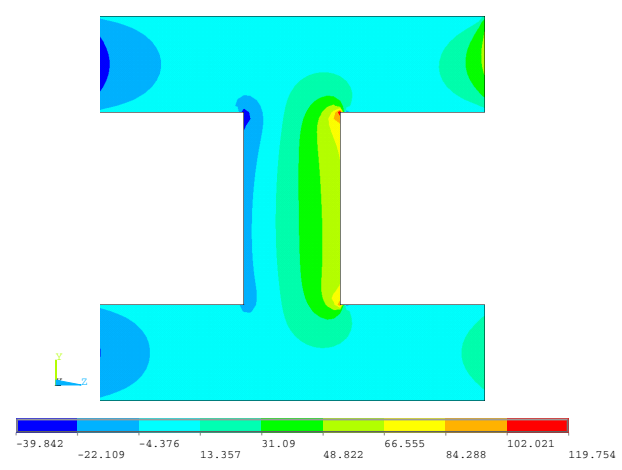

(a)

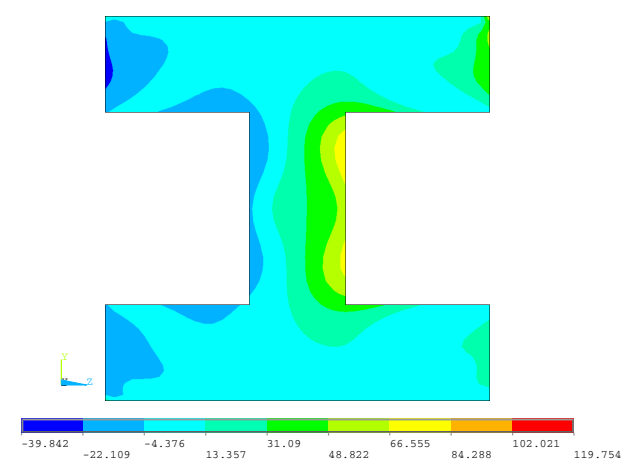

(b)

Fig. 19. Shear stress $\sigma_{x y}[\mathrm{MPa}]$ over the cross-section at $x / l=0$ via (a) FEM 3D-R and (b) PGD FEM 1D (cubic elements, $N=17$ and $N_{c}=7$ ) solutions. Simply supported beam with $l / a=10$. 


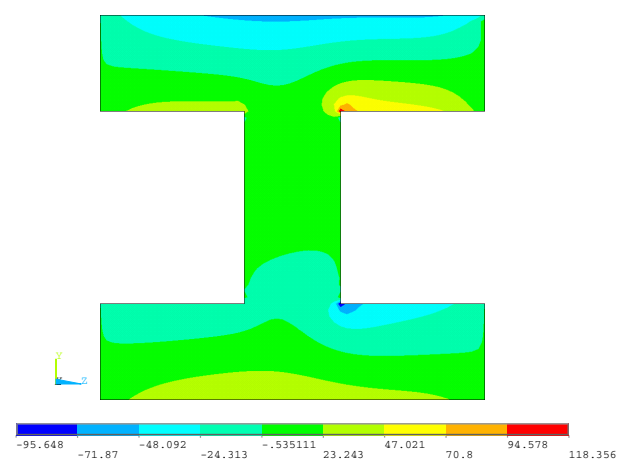

(a)

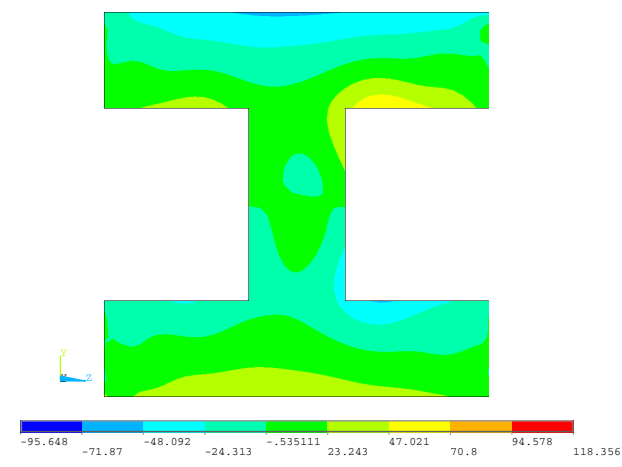

(b)

Fig. 20. Shear stress $\sigma_{x z}[\mathrm{MPa}]$ over the cross-section at $x / l=0$ via (a) FEM 3D-R and (b) PGD FEM 1D (cubic elements, $N=17$ and $N_{c}=7$ ) solutions. Simply supported beam with $l / a=10$.

the beam axis are used ensuring a stress convergence up to two decimal digits, at least. Figures 12-14 present the displacements, whereas the six stress components are shown in Figs. 15-20.

The proposed solution yields a fair accuracy considering that the case under investigation is very challenging: the global behavior is satisfactory matched, whereas the stress concentration at the sharp corners is very difficult to be described even by the three-dimensional finite element solution (stresses increases are the mesh is refined). A possible manner to improve the proposed beam models is the use of a local approximation by means of a layer-wise approach [Carrera et al., 2011].

\section{Conclusion}

The static analysis of three-dimensional beam structures has been carried out by a hierarchical family of one-dimensional beam finite elements derived within a PGD framework. A UF is used to approximate the displacement field over the cross-section. Higher-order models accounting for nonclassical effects (such as shear deformation and in- and out-of-plane warping) have been derived. As far as finite element approximation is concerned, this UF allows to derive finite elements that do not dependent upon the number of nodes per element along the beam axis. Linear, quadratic and cubic Lagrangian approximations have been considered. Thanks to the PGD, the global problem is divided into two coupled problems on the crosssection and along the beam axis. The dimensions of these two problems are smaller than that of an equivalent classical finite element solution. This approach is particular appealing in the case of higher-order solutions with a refined mesh along the beam axis since a high amount of computer volatile memory is required and stuck overflow can occur. Isotropic beams have been investigated. Clamped-clamped and simply supported boundary conditions have been considered. Analyses accounted for both slender and short beams subjected to bending as well as torsional loads. 
Results have been validated through comparison with analytical Navier-type solutions and three-dimensional finite element solutions obtained via the commercial code ANSYS. The presented results showed that quasi three-dimensional solutions for both displacement and stress components can be obtained in a very computational effective manner thanks to the proposed one-dimensional finite element modeling in the framework of the considered variable separation method.

\section{References}

Ammar, A., Mokdad, B., Chinesta, F. and Keunings, R. [2006] "A new family of solvers for some classes of multi-dimensional partial differential equations encountered in kinetic theory modeling of complex fluids," Journal of Non-Newtonian Fluid Mechanics 3(139), 153-176.

Bathe, K. J. [1996] Finite Element Procedures (Prentice Hall, USA).

Berdichevsky, E. A., Armanios, E. and Ashraf, B. [1992] "Theory of anisotropic thin-walled closed-cross-section beams," Composite Engineering 2(5-7), 411-432.

Bognet, B., Bordeu, F., Chinesta, F., Leygue, A. and Poitou, A. [2012] "Advanced simulation of models defined in plate geometries: 3D solutions with $2 \mathrm{D}$ computational complexity," Computer Methods in Applied Mechanics and Engineering 201-205, $1-12$.

Carrera, E. [2003] "Theories and finite elements for multilayered plates and shells: A unified compact formulation with numerical assessment and benchmarking," Archives of Computational Methods in Engineering 10(3), 215-296.

Carrera, E. and Giunta, G. [2009a] "Exact, hierarchical solutions for localized loadings in isotropic, laminated and sandwich shells," Journal of Pressure Vessel Technology 131(4), 041202.

Carrera, E. and Giunta, G. [2009b] "Hierarchical evaluation of failure parameters in composite plates," AIAA Journal 47(3), 692-702.

Carrera, E. and Giunta, G. [2010] "Refined beam theories based on a unified formulation," International Journal of Applied Mechanics 2(1), 117-143.

Carrera, E., Giunta, G., Nali, P. and Petrolo, M. [2010] "Refined beam elements with arbitrary cross-section geometries," Computers and Structures 88(5-6), 283-293.

Carrera, E., Giunta, G. and Petrolo, M. [2011] Beam Structures: Classical and Advanced Theories (John Wiley and Sons, UK).

Castellanos, J. A. and Larrazabal, G. [2013] "A Cholesky out-of-core factorization," Mathematical and Computer Modeling 57(9-10), 2207-2222.

Chinesta, F., Ammar, A., Leygue, A. and Keunings, R. [2011] "An overview of the proper generalized decomposition with applications in computational rheology," Journal of Non-Newtonian Fluid Mechanics 166(11), 578-592.

Gallimard, L., Vidal, P. and Polit, O. [2011] "Coupling finite element and reliability analysis through proper generalized decomposition model reduction," International Journal for Numerical Methods in Engineering 95(13), 1079-1093.

Ganapathi, M., Patel, B. P., Polit, O. and Touratier, M. [1999] "A C ${ }^{1}$ finite element including transverse shear and torsion warping for rectangular sandwich beams," International Journal for Numerical Methods in Engineering 45(1), 47-75.

Giunta, G., Belouettar, S. and Carrera, E. [2010a] "Analysis of FGM beams by means of classical and advanced theories," Mechanics of Advanced Materials and Structures $\mathbf{1 7}(8), 622-635$. 
Giunta, G., Biscani, F., Belouettar, S. and Carrera, E. [2010b] "Analysis of thin-walled beams via a one-dimensional unified formulation through a Navier-type solution," International Journal of Applied Mechanics 3(3), 407-434.

Giunta, G., Biscani, F., Belouettar, S. and Carrera, E. [2011] "Hierarchical modeling of doubly curved laminated composite shells under distributed and localized loadings," Composites: Part B 42(4), 682-691.

Giunta, G., Biscani, F., Belouettar, S., Ferreira, A. J. M. and Carrera, E. [2012] "Free vibration analysis of composite beams via refined theories," Composites Part B: Engineering 44(1), 540-552.

Giunta, G., Crisafulli, D., Belouettar, S. and Carrera, E. [2013] "A thermo-mechanical analysis of functionally graded beams via hierarchical modeling," Composite Structures 95, 676-690.

Giunta, G., Metla, N., Koutsawa, Y. and Belouettar, S. [2013] "Free vibration and stability analysis of three-dimensional sandwich beams via hierarchical models," Composites: Part B 47, 326-338.

Hodges, D. H. [1990] "A review of composite rotor blade modeling," AIAA Journal 28(3), $561-565$.

Horn, R. A. [1990] "The Hadamard product," Proc. of Symposia in Applied Mathematics, Vol. 40, pp. 87-169.

Kant, T. and Manjunath, B. S. [1989] "Refined theories for composite and sandwich beams with $\mathrm{C}^{0}$ finite elements," Computers and Structures 33(3), 755-764.

Kapania, R. K. and Raciti, S. [1989a] "Recent advances in analysis of laminated beams and plates, part I: Shear effects and buckling," AIAA Journal 27(7), 923-934.

Kapania, R. K. and Raciti, S. [1989b] "Recent advances in analysis of laminated beams and plates, part II: Vibrations and wave propagation," AIAA Journal 27(7), 935-946.

Lee, J. [2005] "Flexural analysis of thin-walled composite beams using shear-deformable beam theory," Composite Structures 70(2), 212-222.

Lee, J. and Lee, S. [2004] "Flexural-torsional behavior of thin-walled composite beams," Thin-Walled Structures 42(9), 1293-1305.

Nouy, A. [2010] "A priori model reduction through proper generalized decomposition for solving time-dependent partial differential equations," Computer Methods in Applied Mechanics and Engineering 199(23-24), 1603-1626.

Rand, O. [1998] "Fundamental closed-form solutions for solid and thin-walled composite beams including a complete out-of-plane warping model," International Journal of Solids and Structures 35(21), 2775-2793.

Savoia, M. and Reddy, J. [1992] "A variational approach to three-dimensional elasticity solutions of laminated composite plates," Journal of Applied Mechanics 59(2), 166175.

Stephen, N. G. and Levinson, M. [1979] "A second order beam theory," Journal of Sound and Vibration 67(3), 293-305.

Tornabene, F., Liverani, A. and Caligiana, G. [2012] "Static analysis of laminated composite curved shells and panels of revolution with a posteriori shear and normal stress recovery using generalized differential quadrature method," International Journal of Mechanical Sciences 61(1), 71-87.

Vidal, P., Gallimard, L. and Polit, O. [2012a] "Assessment of a composite beam finite element based on the proper generalized decomposition," Computers and Structures 94(5), 1900-1910.

Vidal, P., Gallimard, L. and Polit, O. [2012b] "Composite beam finite element based on the proper generalized decomposition," Computers and Structures 102-103, 76-86. 
Vidal, P., Gallimard, L. and Polit, O. [2013] "Proper generalized decomposition and layerwise approach for the modeling of composite plate structures," International Journal of Solids and Structures 50(14-15), 2239-2250.

Vidal, P., Gallimard, L. and Polit, O. [2014a] "Explicit solutions for the modeling of laminated composite plates with arbitrary stacking sequences," Composites Part B: Engineering 60, 697-706.

Vidal, P., Gallimard, L. and Polit, O. [2014b] "Shell finite element based on the proper generalized decomposition for the modeling of cylindrical composite structures," Computers and Structures 132, 1-11.

Vidal, P. and Polit, O. [2008] "A family of sinus finite elements for the analysis of rectangular laminated beams," Composite Structures 84(1), 56-72.

Yu, W., Hodges, D. H., Volovoi, V. and Cesnik, C. E. S. [2002] "On Timoshenko-like modeling of initially curved and twisted composites beams," International Journal of Solids and Structures 39(19), 5101-5121. 